\title{
Atomic Structure Variational Calculations in Spectroscopy
}

\author{
Michel R. Godefroid, ${ }^{1 *}$ Per Jönsson ${ }^{2}$ and Charlotte Froese Fischer ${ }^{3}$ \\ ${ }^{1}$ Laboratoire de Chimie Physique Moléculaire, CP160/09, Université Libre de Bruxelles, 50 av. F.D. Roosevelt, B 1050 Bruxelles, Belgium \\ ${ }^{2}$ Department of Physics, Lund Institute of Technology, Box 118 S-22100 Lund, Sweden \\ ${ }^{3}$ Department of Computer Science, Vanderbilt University, Box 1679B, Nashville, TN 37235, U.S.A.
}

Received August 4, 1997; Accepted January 31, 1998

PACS Ref: $31.10 .+z, 31.30 . G s, 32.70 . C s$

\begin{abstract}
Recent $a b$ initio variational calculations of radiative transition probabilities, isotope shifts and hyperfine structures are described in the spirit of the EGAS tradition for plenary talks. A few simple cases are selected to make the exposé at a level accessible to non-specialists in the field and to illustrate how computational atomic structure can be used in atomic spectroscopy for testing theoretical models or experimental results, predicting properties or interpreting them in terms of electron correlation. The effects inherent in the multiconfiguration Hartree-Fock method due to its variational nature are emphasized through some simple analysis of the wave function spatial distribution in correlation with the model used.
\end{abstract}

\section{Introduction}

Atomic structure calculations have always had large impact in spectroscopy. They not only assist the spectroscopist in the analysis of complex spectra by providing some reliable term positions, level designations, synthesis spectra, helping in the assignment of the observed lines, but also contribute to the understanding of the underlying physical processes. The identification of the dominant cascade processes to be considered in the analysis of beam-foil data in order to extract the meanlife from experimental decay curves, is another example of assistance of atomic structure calculations to experimental spectroscopy. Ab initio calculations can also precede observation. A well-known example is the theoretical prediction of the stable negative $\mathrm{Ca}^{-}$ion which was confirmed experimentally. In the spectroscopy of multiply excited states, reliable theoretical data such as energies, widths, lifetimes, autoionization and radiative decay rates, stabilization ratios are essential in the understanding of the experimental spectra. Variational calculations provide a possible way, among others, to face the atomic many-body problem for predicting spectroscopic properties. In the present paper, we select a few examples of applications, hoping to show that these methods are very efficient and accurate and as such, can play an important role in the unceasing interaction between theory and experiment in atomic spectroscopy.

\section{The variational method}

The multiconfiguration Hartree-Fock and Dirac-Fock methods that we are using, can be derived from the variational method [1]. For this reason, the latter is briefly exam-

\footnotetext{
* Research Director the Belgian National Fund for Scientific Research (FNRS).
}

ined though Hartree himself used more his "physical intuition" when he postulated that the central field in which each electron is moving can be determined from the averaged charge distribution of the other electrons [2].

The Rayleigh-Ritz's variation method $[1,3]$ consists of viewing the expectation value of the Hamiltonian as a functional mapping each state $\Psi$ to a real number $E[\Psi]$.

$\langle H\rangle=\frac{\langle\Psi|H| \Psi\rangle}{\langle\Psi \mid \Psi\rangle} \equiv E[\Psi]$.

The state $\Psi$ is an eigenstate of the Hamiltonian $H$ if and only if the total energy $E$ is left unchanged for any infinitesimal variation in the total wave function at the point $\Psi$

$|\Psi\rangle \rightarrow|\Psi+\delta \Psi\rangle \Rightarrow \delta E=0$.

For the ground state (or the lowest of its symmetry), the variational method gives a minimum principle,

$E_{0} \leqslant \frac{\langle\Psi|H| \Psi\rangle}{\langle\Psi \mid \Psi\rangle}$

$E[\Psi]$ being an upper bound to the exact ground state energy $E_{0}$. If we use the following superposition ansatz

$|\Psi\rangle=\sum_{i=1}^{N} c_{i}\left|\Phi_{i}\right\rangle$

for describing the model state using a subspace of $N$ basis states $\Phi_{i}$, the variational parameters $\left\{c_{i}\right\}$ can be determined by solving the generalized eigenvalue problem

$\sum_{j=1}^{N}\left(H_{i, j}-E S_{i, j}\right) c_{j}=0, \quad i=1, \ldots, N$

with

$H_{i, j} \equiv\left\langle\Phi_{i}|H| \Phi_{j}\right\rangle ; \quad S_{i, j} \equiv\left\langle\Phi_{i} \mid \Phi_{j}\right\rangle$.

The total energies can be found as the roots of the secular equations

$\operatorname{det}\left(H_{i, j}-E S_{i, j}\right)=0$.

The use of the $\left\{\Psi^{(k)}\right\}$ eigenfunctions as a model subspace, satisfying

$\left\langle\Psi^{(k)} \mid \Psi^{(l)}\right\rangle=\delta_{k, l} ; \quad\left\langle\Psi^{(k)}|H| \Psi^{(l)}\right\rangle=\varepsilon_{k} \delta_{k, l}$,

i.e. both orthonormality and non-interacting functions, is very useful for the description of excited states since they 
satisfy the following bounding conditions

$E_{i} \leqslant \varepsilon_{i} \quad \forall i=1, \ldots, N$

known as the Hylleraas-Undheim theorem [4]. This theorem tells us that all the approximate eigenvalues obtained by diagonalizing the Hamiltonian in a subspace can only be stabilized when the latter is enlarged.

\section{The non-relativistic MCHF approximation}

The first part of the talk is devoted to non-relativistic calculations in which we are using the non-relativistic Hamiltonian

$H=\sum_{i=1}^{N}\left[-\frac{1}{2} \nabla_{i}^{2}-\frac{Z}{r_{i}}\right]+\sum_{i<j}^{N} \frac{1}{r_{i j}}$.

The atomic wave function is built as a multi-configuration expansion of configuration state functions (CSFs)

$\left|\Psi\left(S M_{S} L M_{L} \pi\right)\right\rangle=\sum_{i} c_{i}\left|\Phi\left(\alpha_{i} S M_{S} L M_{L} \pi\right)\right\rangle$.

Each CSF arising from the configuration (and associated coupling tree) $\alpha_{i}$ is an eigenfunction of the set of spin- and orbital-angular momentum $S^{2}, S_{z}, L^{2}, L_{z}$ and inversion operator $\left(\boldsymbol{r}_{i} \rightarrow-\boldsymbol{r}_{i} ; \forall i\right)$ commuting among each other, together with the non-relativistic Hamiltonian. The associated quantum numbers $S, M_{S}, L, M_{L}, \pi$ define the symmetry of the state. The CSFs are symmetry-adapted linear combinations of Slater-determinants built on a set of oneelectron spin-orbitals

$\phi_{n l m l m_{s}}=\frac{1}{r} P_{n l}(r) Y_{l m_{l} l}(\theta, \varphi) \chi_{m_{s}}$

whose radial distributions $\left\{\boldsymbol{P}_{n l}\right\}$ need to be determined. By applying the variational principle, i.e. searching for the stationarity of the expectation value with respect to any infinitesimal variation in the radial distribution $\delta P_{n l}(r)$, one gets [5] the set of multi-configuration Hartree-Fock equations (MCHF)

$$
\begin{aligned}
\left\{\frac{\mathrm{d}^{2}}{\mathrm{~d} r^{2}}\right. & \left.+\frac{2}{r}\left[Z-Y_{n l}(r)\right]-\varepsilon_{n l, n l}-\frac{l(l+1)}{r^{2}}\right\} P_{n l}(r) \\
& =\frac{2}{r} X_{n l}(r)+\sum_{n^{\prime}} \varepsilon_{n l, n^{\prime} l} P_{n^{\prime} l}(r)
\end{aligned}
$$

for the unknown radial distributions $P_{n l}(r)$. These integrodifferential equations are coupled to each other through the direct $(Y)$ and exchange $(X)$ potentials and through the radial orthogonality constraints within the same $l$ subspace. The configuration mixing coefficients $\left\{c_{i}\right\}$ appearing in (9) also enter in the explicit form of the potentials and can be determined by solving the configuration interaction (CI) problem for the current set of radial distributions

$H C=C E$,

associated with the application of the variation method to the multiconfiguration expansion (9). The MCHF and CI problems are solved iteratively until self-consistency is reached for the radial distributions and for the selected CIeigenvector.

\section{On the dependence of the one-electron orbitals}

The fact that the multi-configuration Hartree-Fock method that we use results from the application of the variational method often makes the MCHF wave function solution of the problem, strongly dependent on the energy functional used to derive the MCHF equations. This will be illustrated in quite different situations through realistic examples, giving us the opportunity to explain at the same time how the MCHF method can be used for describing specific physical effects. The direct influence of the optimization scheme on the shape of the resulting one-electron orbitals in MCHF variational calculations can be taken advantageously for describing some specific effect but could also bring some undesirable (and sometimes unexpected) distortion of the wave function for the property of interest. Any user of variational methods should be aware of these facts.

A well-known simple example is the term-dependence of the radial distributions. Let us consider the four-electron beryllium excited configuration $1 s^{2} 2 s 2 p$ from which the two ${ }^{1,3} \mathrm{P}^{\mathrm{o}}$ states arise. The $\left(M_{L}, M_{S}\right)=(1,0)$ subspace is spanned by two Slater determinants

\section{$\left|1 \mathrm{~s} \overline{1 s 2 s} 2 p_{+1}\right|$ and $\left|1 \mathrm{~s} \bar{s} 2 \mathrm{~s} \overline{2 p}_{+1}\right|$}

where the bar/unbar notation is used for specifying the $m_{s}=-1 / 2$ or $+1 / 2$ spin projection and the subscript corresponds to the $m_{l}$ value. The two $\boldsymbol{L}^{2}$ and $\boldsymbol{S}^{2}$ eigenstates correspond to the orthogonal linear combinations

$$
\begin{aligned}
& \left|\Psi\left({ }^{3} \mathrm{P}_{1,0}^{\mathrm{o}}\right)\right\rangle=\frac{1}{\sqrt{2}}\left\{\left|1 \mathrm{~s} \overline{\mathrm{s} 2 \mathrm{~s}} 2 \mathrm{p}_{+1}\right|+\left|1 \mathrm{~s} \overline{\mathrm{s}} 2 \mathrm{~s} 2 \mathrm{p}_{+1}\right|\right\} \\
& \left|\Psi\left({ }^{1} \mathrm{P}_{1,0}^{\mathrm{o}}\right)\right\rangle=\frac{1}{\sqrt{2}}\left\{\left|1 \mathrm{~s} \overline{\mathrm{s} 2 \mathrm{~s}} 2 \mathrm{p}_{+1}\right|-\left|1 \mathrm{~s} \overline{\mathrm{s}} 2 \mathrm{~s} \overline{\mathrm{p}}_{+1}\right|\right\} .
\end{aligned}
$$

The \pm sign of the linear combination is crucial though manifesting itself in the total energy expressions only through the angular coefficient of the exchange $G^{1}(2 \mathrm{~s}, 2 \mathrm{p})$ Slater integral involving the two open shells $2 \mathrm{~s}$ and $2 \mathrm{p}$,

$$
\begin{aligned}
E\left({ }^{3,1} \mathrm{P}^{\mathrm{o}}\right)= & 2 I_{1 \mathrm{~s}}+I_{2 \mathrm{~s}}+I_{2 \mathrm{p}}+F^{0}(1 \mathrm{~s}, 1 \mathrm{~s})+2 F^{0}(1 \mathrm{~s}, 2 \mathrm{~s}) \\
& -G^{0}(1 \mathrm{~s}, 2 \mathrm{~s})+2 F^{0}(1 \mathrm{~s}, 2 \mathrm{p})-\frac{1}{3} G^{1}(1 \mathrm{~s}, 2 \mathrm{p}) \\
& +F^{0}(2 \mathrm{~s}, 2 \mathrm{p}) \mp \frac{1}{3} G^{1}(2 \mathrm{~s}, 2 \mathrm{p}) .
\end{aligned}
$$

In this one-configuration Hartree-Fock approximation, the radial orbitals resulting from the two separate optimizations differ substantially from each other. As can be seen from Fig. 1 , the $2 p$ radial distribution of the ${ }^{1} \mathrm{P}^{\mathrm{o}}$ state is more diffuse than that of the ${ }^{3} \mathrm{P}^{\mathrm{o}}$ state. The difference between term-dependent and configuration-average results can be regarded as higher contributions in a perturbation expansion based on the latter [6]. In variational calculations however, it would be a pity to use the $2 p$ function arising from the ${ }^{1} \mathrm{P}^{\mathrm{o}}$ Hartree-Fock optimization (or from an average calculation) for describing the ${ }^{3} \mathrm{P}^{0}$ state.

Another situation revealing a natural orbital differentiation occurs in the two-configuration description of the 
Term dependence

Be 1s2.2s.2p (3P,1P)

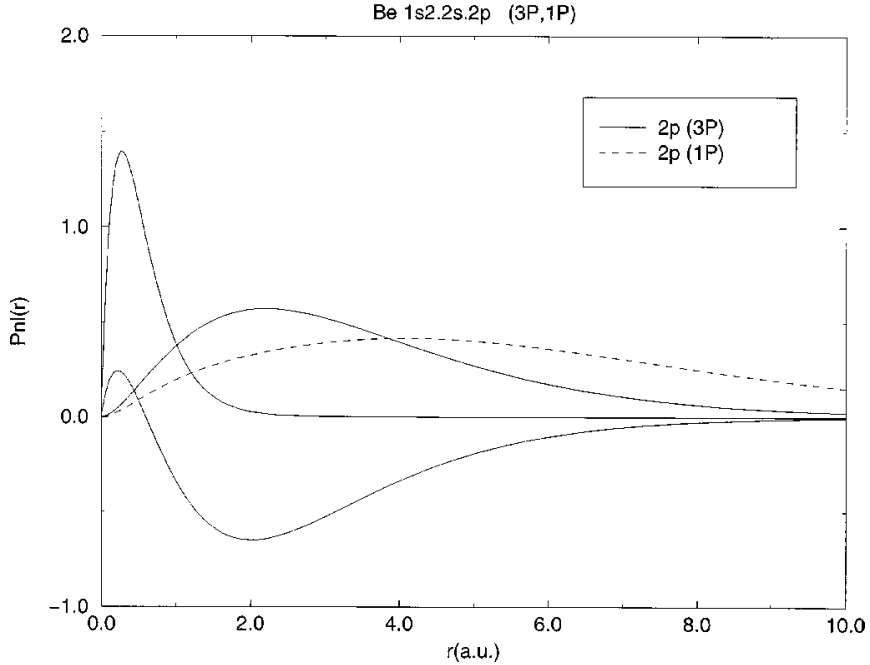

Fig. 1. Term-dependence for $\mathrm{Be} 1 \mathrm{~s}^{2} 2 \mathrm{~s} 2 \mathrm{p}^{3,1} \mathrm{P}^{\mathrm{o}}$. The $2 \mathrm{p}$ radial distribution of the ${ }^{1} \mathrm{P}^{\mathrm{o}}$ state is more diffuse than that of the ${ }^{3} \mathrm{P}^{\mathrm{o}}$ state. The (common) inner $1 \mathrm{~s}$ (no node) and 2s (one node) orbitals are also displayed on the same figure.

lowest ${ }^{1} \mathrm{P}^{\mathrm{o}}$ state of beryllium that one would use for improving the Hartree-Fock approximation for this state,

$|\Psi\rangle^{\mathrm{HF}}=\left|1 \mathrm{~s}^{2} 2 \mathrm{~s} 2 \mathrm{p}^{1} \mathrm{P}^{\mathrm{o}}\right\rangle ; \quad E=-14.394735$ a.u.

by allowing for the mixing with the close configuration $2 \mathrm{p} 3 \mathrm{~d}$ $|\Psi\rangle^{\mathrm{MCHF}}=0.97524\left|1 \mathrm{~s}^{2} 2 \mathrm{~s} 2 \mathrm{p}^{1} \mathrm{P}^{\mathrm{o}}\right\rangle-0.22116\left|1 \mathrm{~s}^{2} 2 \mathrm{p} 3 \mathrm{~d}^{1} \mathrm{P}^{\mathrm{o}}\right\rangle$ $E=-14.411563$ a.u.

In this approach, the $2 p$ orbital plays the double rôle of spectroscopic and correlation orbital. If we use indeed a nonorthogonal scheme allowing the two $2 \mathrm{p}$ orbitals to be $a$ priori different, we get the following wave function and corresponding total energy

$|\Psi\rangle^{\mathrm{MCHF}}=0.97159\left|1 \mathrm{~s}^{2} 2 \mathrm{~s} 2 \mathrm{p}^{1} \mathrm{P}^{\mathrm{o}}\right\rangle-0.23665\left|1 \mathrm{~s}^{2} 2 \mathrm{p}^{\prime} 3 \mathrm{~d}^{1} \mathrm{P}^{\circ}\right\rangle ;$

$E=-14.418786$ a.u.

with a large energy stabilization effect relatively to the above orthogonal description. Figure 2 beautifully illustrates the different shapes of the $2 p$ and $2 p^{\prime}$ orbitals, the first describing the spectroscopic state $2 \mathrm{~s} 2 \mathrm{p}^{1} \mathrm{P}^{\mathrm{o}}$ while the second, together with the $3 \mathrm{~d}$ orbital, optimize the correlation interaction.

A third example of the "variational principle in action" is given by the analysis of the correlation orbitals in the pair correlation picture. Let us consider the ground state of beryllium which is described by the best single Slater determinant

$|\Psi\rangle^{\mathrm{HF}}=\left|1 \mathrm{~s}^{2} 2 \mathrm{~s}^{2}{ }^{1} \mathrm{~S}\right\rangle ; \quad E=-14.573023$ a.u.

in the Hartree-Fock approximation. Taking the $n=4$ orbital active set made of all possible orbitals $[0 \leqslant l \leqslant(n-1)]$ up to $n=4$, i.e.

$\{1 \mathrm{~s}, 2 \mathrm{~s}, 2 \mathrm{p}, \ldots, 3 \mathrm{~d}, 4 \mathrm{~s}, 4 \mathrm{p}, 4 \mathrm{~d}, 4 \mathrm{f}\}$

the valence correlation wave function is described by considering all possible excitations of the valence electron pair
The non-orthogonal approach

$\mathrm{Be}\left(2 \mathrm{~s} 2 \mathrm{p}+2 \mathrm{p}^{\prime} 3 \mathrm{~d}\right) 1 \mathrm{P}$

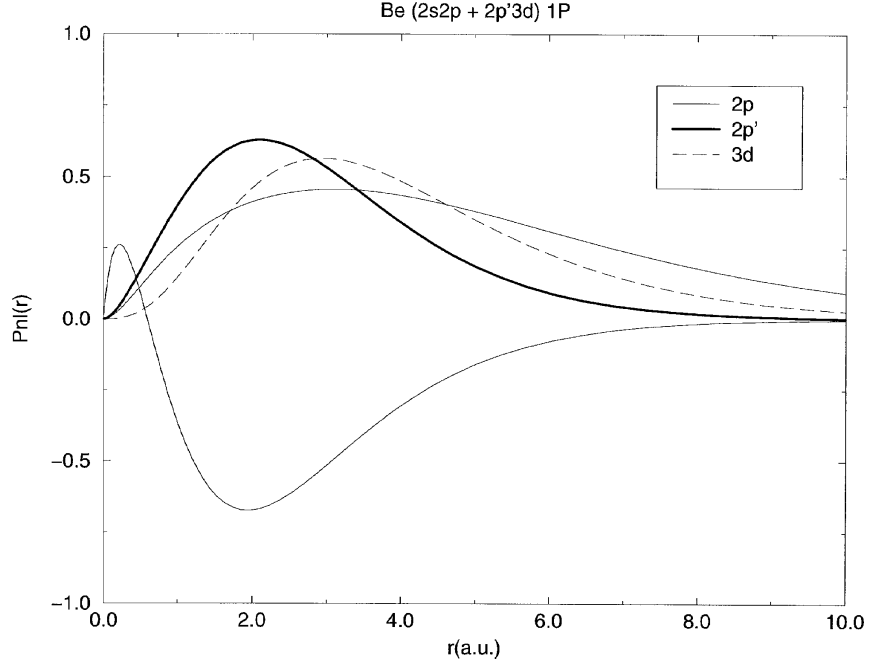

Fig. 2. The non-orthogonal approach for $\operatorname{Be}\left\{1 \mathrm{~s}^{2} 2 \mathrm{~s} 2 \mathrm{p}+1 \mathrm{~s}^{2} 2 \mathrm{p}^{\prime} 3 \mathrm{~d}^{1} \mathrm{P}^{\mathrm{o}}\right\}$ : the $2 \mathrm{p}$ orbital describes the spectroscopic state $2 \mathrm{~s} 2 \mathrm{p}^{1} \mathrm{P}^{\mathrm{o}}$ while the $2 \mathrm{p}^{\prime}$ orbital together with $3 \mathrm{~d}$, optimize the correlation interaction. The $2 \mathrm{~s}$ orbital (one node) is also displayed.

$E=-14.618990$ a.u.

The core-valence correlation wave function is obtained by "promoting" one core electron together with one valence electron

$|\Psi\rangle^{\mathrm{CV}}=\left|1 \mathrm{~s}^{2} 2 \mathrm{~s}^{2}{ }^{1} \mathrm{~S}\right\rangle+\sum_{n l n^{\prime} l} b_{n l n^{\prime} l}\left|1 \mathrm{~s} 2 \operatorname{snn}^{\prime} l^{\prime} l^{1} \mathrm{~S}\right\rangle$

$E=-14.578782$ a.u.

The core correlation MCHF calculation takes into account the correlatin within the core,

$|\Psi\rangle^{\mathrm{C}}=\left|1 \mathrm{~s}^{2} 2 \mathrm{~s}^{2}{ }^{1} \mathrm{~S}\right\rangle+\sum_{n l n^{\prime} l} c_{n l n^{\prime} l}\left|2 \mathrm{~s}^{2} n l n^{\prime} l^{1} \mathrm{~S}\right\rangle$

$E=-14.614398$ a.u.

Each of the above sums represents correlation between a particular pair of electrons, namely $(2 \mathrm{~s}, 2 \mathrm{~s})$ for valence correlation, $(1 \mathrm{~s}, 2 \mathrm{~s})$ for core-valence, and $(1 \mathrm{~s}, 1 \mathrm{~s})$ for core correlation. For this reason the sums are referred to as "pair correlation functions". In the present case there is one pair correlation function for each type of correlation, but in more complex situations, each type of correlation may involve many different pair correlation functions. The dependence of the orbitals on the type of correlation considered is illustrated by the sequence of Fig. 3(a)-(c) which reveal the collapse of correlation orbitals when going from a valence- to a core-correlation calculation. Unfortunately, correlation effects are not additive and interference effects do occur through multiple excitations. This example only shows that it is indeed possible to target a given type of correlation by modelling the multiconfiguration expansion. However, the resulting set of radial distributions is so welltailored thanks to the "variational principle in action" that 

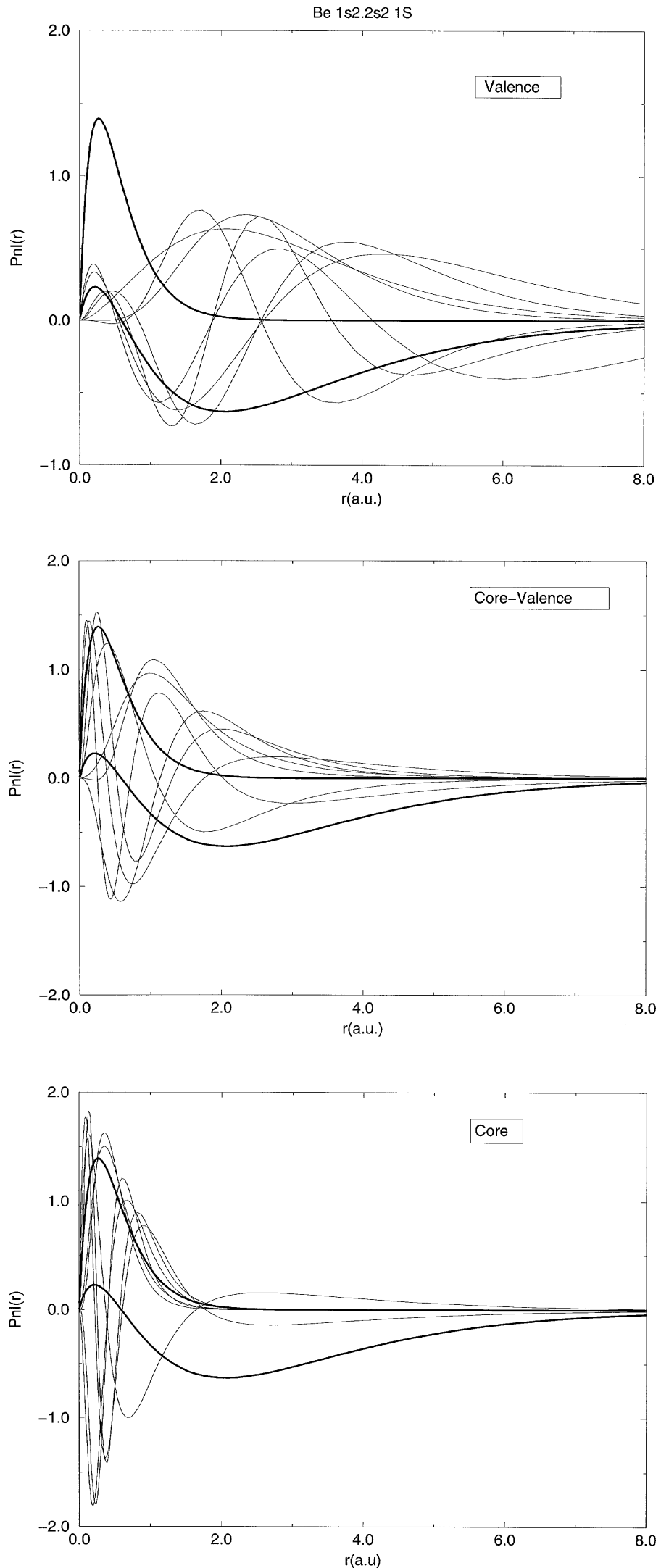

Fig. 3. (a)-(c) Collapse of the correlation orbitals from valence, corevalence and core correlation MCHF calculations of $\mathrm{Be} 1 \mathrm{~s}^{2} 2 \mathrm{~s}^{2}{ }^{1} \mathrm{~S}$. The two thick solid lines correspond to the spectroscopic $1 \mathrm{~s}$ (no node) and $2 \mathrm{~s}$ (one node) orbitals. The other lines represent the radial distributions of the correlation orbitals of the $n=4$ active set.

it quickly becomes inappropriate to represent another type of correlation. In our four-electron example, it should be clear that using core-correlation orbitals for describing valence correlation is hopeless. The situation becomes obviously worse when considering larger systems, with the space-extension of the atom being on a par with the increasing number of electrons.

\section{Some applications of the Active Space Method}

\subsection{Allowed transitions in $\mathrm{Li} \mathrm{I}, \mathrm{NaI}$ and Be I}

The active space method consists of generating CSF lists by electron excitation from the reference configuration to an active set of orbitals (AS). The latter can be increased in a systematic way, allowing the convergence of the property to be carefully studied. The "complete active space" (CAS) is spanned by all CSFs of a particular symmetry which can be generated from a given active set of orbitals. For instance, the $n=3$ CAS space describing a three-electron atom or ion and resulting from the active set $\{1 \mathrm{~s}, 2 \mathrm{~s}, 2 \mathrm{p}, 3 \mathrm{~s}, 3 \mathrm{p}, 3 \mathrm{~d}\}$ contains $27 \mathrm{CSF}$ of ${ }^{2} \mathrm{~S}$ symmetry and even parity,

$$
\left\{1 \mathrm{~s}^{2} 2 \mathrm{~s}, 1 \mathrm{~s} 2 \mathrm{~s}\left({ }^{1} \mathrm{~S}\right) 3 \mathrm{~s}, 1 \mathrm{~s} 2 \mathrm{~s}\left({ }^{3} \mathrm{~S}\right) 3 \mathrm{~s}, \ldots, 3 \mathrm{~s} 3 \mathrm{~d}^{2}\left({ }^{1} \mathrm{~S}\right), 3 \mathrm{p}^{2}\left({ }^{1} \mathrm{D}\right) 3 \mathrm{~d}^{2} \mathrm{~S}\right\} \text {. }
$$

A first illustration of the active space method is given by the study of the radiative transition probability of the $2 s-2 p$ resonance line of lithium using independently optimized CAS expansions [7]. The total energy and number of configuration state functions (NCSF) are reported in Table I for increasing active sets. The active set is specified in all the tables by the principal quantum number value $n$. The notation $9 k$ indicates that the $l$-restriction $l \leqslant l_{\max }=7$ ( $k$-electron) is used. The oscillator strength can be then monitored as a function of the orbital active set specified by the maximum principal quantum number. Figure 4 shows the convergence of the length and velocity forms of the oscillator strengths to each other, but also to the highly accurate results of Yan and Drake [8] who used three-electron Hylleraas wave functions of the type

$$
\begin{aligned}
\Psi\left(\boldsymbol{r}_{1}, \boldsymbol{r}_{2}, \boldsymbol{r}_{3}\right)= & \mathscr{A} \sum_{t} \sum_{\mu_{t}} a_{t, \mu_{t}} \Phi_{t, \mu_{t}}\left(\alpha_{t}, \beta_{t}, \gamma_{t}\right) \\
& \times(\text { angular function)(spin function) }
\end{aligned}
$$

with

$\Phi_{t, \mu_{t}}\left(\alpha_{t}, \beta_{t}, \gamma_{t}\right)=r_{1}^{j_{1}} r_{2}^{j_{2}} r_{3}^{j_{3}} r_{12}^{j_{1} 2} r_{23}^{j_{2}} r_{31}^{j_{31}} \mathrm{e}^{-\alpha_{t} r_{1}-\beta_{t} r_{2}-\gamma_{t} r_{3}}$

Table I. CAS-MCHF calculations for $1 s^{2} 2 s^{2} S$ and $1 s^{2} 2 p^{2} P^{o}$ in LiI (from ref. [7]). Total energies (E) and number of configuration states (NSCF) for different active sets $(A S)$

\begin{tabular}{clrllr}
\hline & \multicolumn{1}{l}{$1 \mathrm{~s}^{2} 2 \mathrm{~s}^{2} \mathrm{~S}$} & & $1 \mathrm{~s}^{2} 2 \mathrm{p}^{2} \mathrm{P}^{\mathrm{o}}$ \\
\cline { 2 - 3 } \cline { 5 - 6 } AS & $E$ (a.u.) & NCSF & & $E$ (a.u.) & NCSF \\
\hline HF & -7.4327269 & 1 & & -7.3650697 & 1 \\
2 & -7.4545653 & 4 & & -7.3801230 & 5 \\
3 & -7.4731843 & 27 & & -7.4045877 & 44 \\
4 & -7.4762030 & 110 & & -7.4078510 & 205 \\
5 & -7.4771599 & 338 & & -7.4091670 & 690 \\
6 & -7.4775797 & 866 & & -7.4096440 & 1880 \\
7 & -7.4777724 & 1948 & & -7.4098569 & 4424 \\
8 & -7.4778745 & 3974 & & -7.4099656 & 9319 \\
$9 \mathrm{k}$ & -7.4779244 & 7240 & & -7.4100181 & 17330 \\
$10 \mathrm{k}$ & -7.4779508 & 12054 & & -7.4100460 & 29261 \\
$11 \mathrm{k}$ & -7.4779657 & 18724 & -7.4100616 & 45916 \\
\hline
\end{tabular}




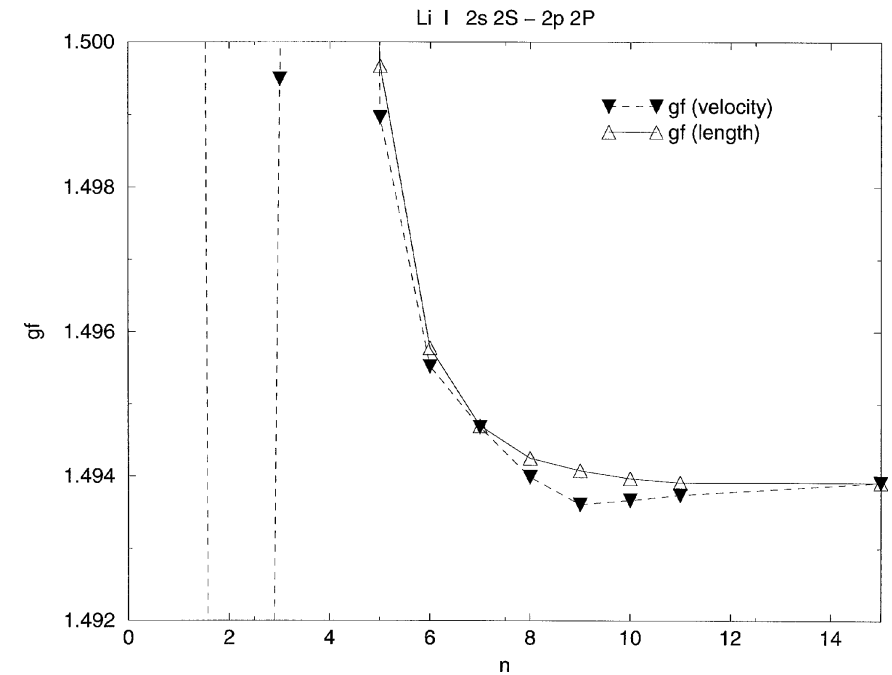

Fig. 4. Convergence of the length and velocity forms of the oscillator strengths $(g f)$ for $2 \mathrm{~s}^{2} \mathrm{~S}-2 \mathrm{p}^{2} \mathrm{P}^{\mathrm{o}}$ in LiI as a function of the orbital active set specified by the principal quantum number $n$. The converged value correspond to the infinite nuclear mass result $g f_{l}=g f_{v}=1.493914$ of Yan and Drake [8].

$\mu_{t}$ denotes a sextuple of integer powers $j_{1}, j_{2}, j_{3}, j_{12}, j_{23}$ and $j_{31}$ and the index $t$ labels different set of nonlinear parameters $\left\{\alpha_{t}, \beta_{t}, \gamma_{t}\right\}$. In their variational approach, the interelectronic distances appear explicitly and a complete optimization is performed with respect to all the nonlinear parameters. This representation gives extremely accurate wave functions and properties, as proven by the impressive convergence of length and velocity forms (for infinite nuclear mass)

$f_{\text {length }}=0.7469572(10) ; \quad f_{\text {velocity }}=0.7469571(54)$

bringing a definitive confirmation of all the previous variational calculations [8]. The problem of the longstanding disagreement between theory and observation $[7,9]$ has been solved by new beam-gas-dye laser spectroscopy measurements of Volz and Schmoranzer [10] which are confirmed by Schmitt $e$ t al's work at the present EGAS meeting with a higher precision [11] and by the photoassociative spectroscopy measurement of McAlexander et al. with extremely small error bars [12].

The resonance line of sodium also suffered for a long time from a systematic disagreement between theory and experiment [7,9]. As for LiI, the last experimental values from beam-gas-laser spectroscopy $[10,13]$ or from high precision linewidth measurement of laser-cooled atoms [14] are in excellent agreement with the most recent $a b$ initio variational calculations using the active space MCHF method [7, 15]. This happy ending for both LiI and Na I should not incite us to pass the recent paper by Curtis et al. [18] in silence. From an analysis of the effect of a time-dependent distorsion term in the intensity decay law

$I(t)=I(0) \exp \left(-\frac{t}{\tau_{k}}\right)+C f(t)$,

they indeed concluded that "deviations from the validity of the exponential law that are too small to be directly detected could lead to inaccuracies in the value of the extracted meanlives that are significant at this level of precision."
The size of a CAS expansion grows dramatically with the number of electrons for a given orbital active set and it is well known that many configurations have very small expansion coefficients, contributing little to the total energy or to the studied property [19]. Different strategies can be used to keep the size of the MCHF and CI expansions manageable. Four-electron systems are very useful from a pedagogical point of view for explaining such approaches. In Table II are reported the sizes of different CSF expansions describing the $1 \mathrm{~s}^{2} 2 \mathrm{~s} 2 \mathrm{p}^{1} \mathrm{P}^{\mathrm{o}}$ of berylium-like ions for the $n=9$ orbital active set

$\{1 \mathrm{~s}, 2 \mathrm{~s}, 2 \mathrm{p}, \ldots, 9 \mathrm{~s}, 9 \mathrm{p}, 9 \mathrm{~d}, \ldots, 91\}$.

A valence-correlation (V) model would keep the 1s shell inactive. A calculation which considers core-valence (CV) excitations would keep one of the four electrons in the 1s orbital while a CAS expansion would include all types of excitations taking the core-correlation (C) into account. As can be seen from the number of CSFs, the size of the multiconfiguration expansion grows rapidly. By specifying that at least two orbitals have principal quantum numbers $n \leqslant m$, it is possible to reduce considerably the sizes of the MCHF expansions comparatively to the CAS wave function, hoping to keep the major physical effects, i.e. all the valence correlation with the dominant core-valence and core-excitations. One can evaluate the effect of the constraints imposed by following the property for different "limited population" (LP) thresholds $m$, the $m=n_{\max }$ case associated to the orbital active set restoring the original CAS expansion. Such an example is given in Table III for the oscillator strength of the resonance line of $\mathrm{BeI}$ for the largest active sets [20].

Table II. Size of $M C H F$ expansions for $1 s^{2} 2 s 2 p^{1} P^{o}$ using the $n=9$ orbital active set for the valence $(V)$, core-valence $(C V)$ and core $(C)$ correlation models. For the latter, different "limited population" $(L P)$ constraints are used for generating the configuration expansion (see text)

\begin{tabular}{lllr}
\hline Model & & Constraints & NCSF \\
\hline $\mathrm{V}$ & $1 \mathrm{~s}^{2} n n^{\prime} l^{\prime}$ & - & 232 \\
$\mathrm{CV}+\mathrm{V}$ & $1 \operatorname{s}^{\prime} n^{\prime} l^{\prime} n^{\prime \prime} l^{\prime \prime}$ & - & 17826 \\
$\mathrm{C}+\mathrm{CV}+\mathrm{V}$ & $\operatorname{nn}^{\prime} l^{\prime} n^{\prime \prime} l^{\prime \prime} n^{\prime \prime} l^{\prime \prime \prime}$ & $-(\mathrm{CAS})$ & 751736 \\
& & $\min 2(n \leqslant 3)$ & 25102 \\
& & $\min 2(n \leqslant 4)$ & 84667 \\
\hline
\end{tabular}

Table III. SDTQ-LP-MCHF gf-values of $2 s^{2}{ }^{1} S-2 s 2 p^{1} P^{o}$ in Be I (from ref. [20]). Transition energy $(\Delta E)$, length $\left(g f_{l}\right)$ and velocity $\left(g f_{v}\right)$ oscillator strengths, for a given active set $(A S)$ and associated limited population constraint (see text and footnote), with the corresponding numbers of configuration state functions (NCSF)

\begin{tabular}{lclll}
\hline AS & NCSF $\left({ }^{1} \mathrm{~S}\right) /\left({ }^{1} \mathrm{P}^{\mathrm{o}}\right)$ & $\Delta E($ a.u. $)$ & $g f_{l}$ & $g f_{v}$ \\
\hline $8^{\mathrm{a}}$ & $6865 / 16298$ & 0.1943140 & 1.37550 & 1.37605 \\
$8^{\mathrm{b}}$ & $21055 / 53400$ & 0.1941996 & 1.37476 & 1.37604 \\
$9^{\mathrm{a}}$ & $10417 / 25102$ & 0.1941834 & 1.37519 & 1.37594 \\
$9^{\mathrm{b}}$ & $33298 / 84667$ & 0.1940675 & 1.37445 & 1.37589 \\
recommended $[20]$ & & \multicolumn{2}{c}{$1.375(2)$} \\
\hline
\end{tabular}

${ }^{a}$ min. 2 electrons with $n \leqslant 3$ (MCHF).

${ }^{\mathrm{b}}$ min. 2 electrons with $n \leqslant 4(\mathrm{CI})$. 
Single, double, triple and quadruple (SDTQ) excitations have been included using the LP constraint "minimum two electrons with $n \leqslant 3$ " for the MCHF calculations. The LPthreshold $m$ is increased from 3 to 4 in subsequent CI calculations. Figure 5 illustrates the smooth convergence of the oscillator strengths calculated in the length and velocity formalisms. The accuracy of the final recommended value $1.375(2)$ is estimated from the analysis of the convergence trends. These calculations, together with similar work on B II [21] and with a detailed comparison with other variational calculations $[22,23]$, revealed that the isoelectronic smoothing of beam-foil data $[24,25]$ produced line strengths definitely too low for the neutral end of the sequence and that the accuracy of previous variational Hylleraas calculations [26] was overestimated. The reader is referred to the original references $[20,23]$ for more details.

Another strategy commonly used for limiting the rapid increase in the size of the wave function expansion is the multi-reference MCHF (or CI) approach. The concept is rather easy to understand. Pair-correlation functions are obtained through single and double replacements (SD) from the different pairs of electrons in a reference set. The latter usually contains the main correlation contributors. A classical example is the beryllium ground state for which the reference set can be defined by the "near-degeneracy" Layzer complex [27]

$\left\{1 \mathrm{~s}^{2} 2 \mathrm{~s}^{2}+1 \mathrm{~s}^{2} 2 \mathrm{p}^{2}{ }^{1} \mathrm{~S}\right\}$.

Using the $n=3$ orbital active set for generating the SDexpansions from the mono-reference $1 \mathrm{~s}^{2} 2 \mathrm{~s}^{2}{ }^{1} \mathrm{~S}$, one gets a multiconfiguration expansion of $19 \mathrm{CSFs}$. Using the same orbital active set, the SD expansion obtained from the above $t w o$-configuration reference set contains 37 CSFs. A comparison of the two lists shows that the $(37-19)=18$ "extra" CSFs are triple or quadruple excitations with respect to the $1 \mathrm{~s}^{2} 2 \mathrm{~s}^{2}$ component. So, the SD-multireference approach is a way of taking the effect of higher excitations into account.

This method has been used for a systematic study of the $2 \mathrm{~s}^{2}{ }^{1} \mathrm{~S}-2 \mathrm{~s} 3 \mathrm{p}^{1} \mathrm{P}^{\mathrm{o}}$ transition in the beryllium sequence

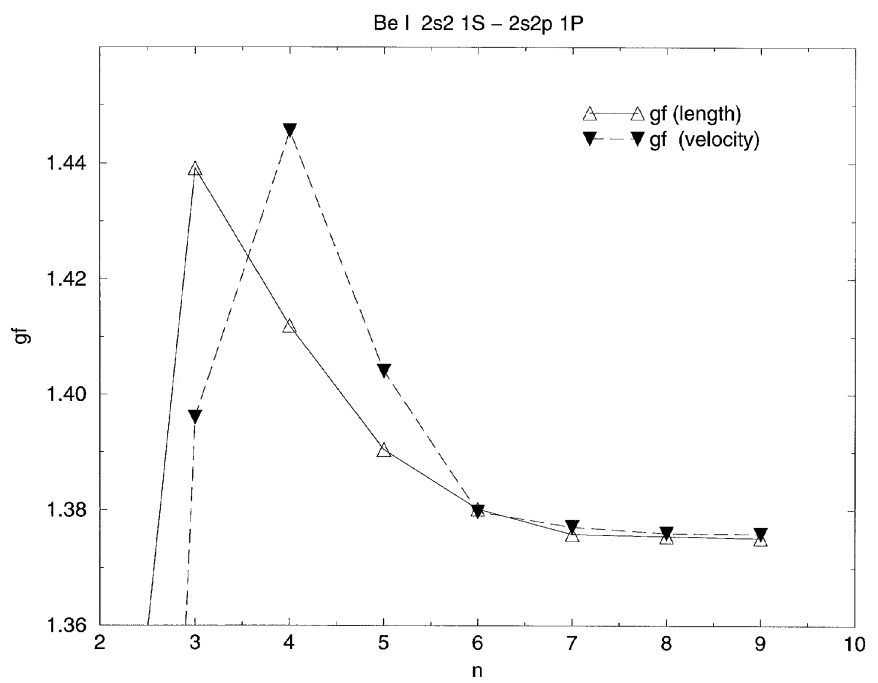

Fig. 5. Convergence of the length and velocity forms of the oscillator strengths $(g f)$ for $2 \mathrm{~s}^{2}{ }^{1} \mathrm{~S}-2 \mathrm{~s} 2 \mathrm{p}^{1} \mathrm{P}^{\mathrm{o}}$ in $\mathrm{Be} \mathrm{I}$ as a function of the orbital active set.
$(4 \leqslant Z \leqslant 10)[28]$ using the two following reference sets $1 \mathrm{~s}^{2}\left\{2 \mathrm{~s}^{2}, 2 \mathrm{p}^{2}\right\}{ }^{1} \mathrm{~S}$ and $1 \mathrm{~s}^{2}\{2 \mathrm{~s} 2 \mathrm{p}, 2 \mathrm{~s} 3 \mathrm{p}, 2 \mathrm{p} 3 \mathrm{~s}, 2 \mathrm{p} 3 \mathrm{~d}\}{ }^{1} \mathrm{P}^{\mathrm{o}}$.

Neutral Be I is a difficult case due to extensive cancellations in the calculation of the electric dipole transition element. Table IV shows how the transition data converge for the three models of correlation. The notation "rm" designates that the relatistic-shift (" $r$ ") and mass-polarization (" $\mathrm{m}$ ") corrections have been included by a rediagonalization of the interaction matrix. The difference in length and velocity results is clearly reduced when going beyond the valence (outer) correlation model. Though the full-correlation calculations have not completely converged, they can be easily extrapolated. The agreement with the calculations of Chung and Zhu [29] $\left(g f_{l}=0.00914\right.$ and $\left.g f_{v}=0.00907\right)$ can be considered as excellent in comparison with earlier theories that differed by factors $2-3$.

\subsection{Isotope shifts in BI}

An introduction to isotope shifts in atomic spectra can be found in King [30]. For the present paper, we only need to remember the effect of the separation of the centre of mass coordinates for a $N$-electron atom [31]. Besides the normal mass shift (NMS) correction which can be calculated exactly from the observed transition energy, the specific mass shift (SMS) can be calculated as the first order correction of the polarization term

$E_{\mathrm{sms}}=-\left\langle\Psi\left|\frac{1}{M} \sum_{i<j}^{N} \nabla_{i} \cdot \nabla_{j}\right| \Psi\right\rangle \equiv \frac{1}{M} S_{\mathrm{sms}}$,

for both levels involved in a transition.

Table IV. Transition energies $(\Delta E)$, length line strengths $\left(S_{l}\right)$, and oscillator strengths $\left(g f_{l}\right.$ for length and $g f_{v}$ for velocity formalisms) for the $\left(2 s^{2}{ }^{1} S-2 s 3 p^{1} P^{o}\right)$ transition of BeI, using different active sets $(A S)$ and correlation models (from ref. [28])

\begin{tabular}{llll}
\hline AS & $\Delta E$ (a.u.) & $S_{l}$ (a.u.) & $g f_{l} / g f_{v}$ \\
\hline $\begin{array}{lll}\text { Outer Correlation } \\
3\end{array}$ & & & \\
4 & 60219 & 0.105682 & $0.019331 / 0.021702$ \\
5 & 60151 & 0.076661 & $0.014007 / 0.016349$ \\
6 & 60141 & 0.071330 & $0.013031 / 0.015418$ \\
& 60151 & 0.069579 & $0.012711 / 0.015245$
\end{tabular}

Core-valence and Outer Correlation

\begin{tabular}{|c|c|c|c|}
\hline 5 & 60298 & 0.058227 & $0.010665 / 0.010401$ \\
\hline 6 & 60246 & 0.052872 & $0.009676 / 0.009483$ \\
\hline 7 & 60241 & 0.051224 & $0.009373 / 0.009286$ \\
\hline $8 \mathrm{i}$ & 60237 & 0.050524 & $0.009245 / 0.009175$ \\
\hline $9 \mathrm{i}$ & 60236 & 0.050196 & $0.009185 / 0.009141$ \\
\hline $10 \mathrm{i}$ & 60235 & 0.050097 & $0.009166 / 0.009143$ \\
\hline 10ir & 60243 & 0.054685 & $0.009235 / 0.009462$ \\
\hline \multicolumn{4}{|c|}{ Full Correlation } \\
\hline 6 & 60279.4 & 0.057889 & $0.010600 / 0.010261$ \\
\hline 7 & 60200.5 & 0.052610 & $0.009620 / 0.009480$ \\
\hline $8 \mathrm{i}$ & 60181.3 & 0.050634 & $0.009256 / 0.009093$ \\
\hline $9 \mathrm{i}$ & 60181.2 & 0.050029 & $0.009145 / 0.008974$ \\
\hline $10 \mathrm{i}$ & 60181.6 & 0.049286 & $0.009009 / 0.008883$ \\
\hline $11 \mathrm{i}$ & 60181.5 & 0.048856 & $0.008931 / 0.008834$ \\
\hline $11 \mathrm{ir}$ & 60190.6 & 0.049248 & $0.009004 / 0.008901$ \\
\hline 11 irm & 60189.8 & 0.049133 & $0.008983 / 0.008878$ \\
\hline Extrap. & 60181.5 & & $0.008826 / 0.008777$ \\
\hline Extrap.(rm) & 60189.8 & & $0.008878 / 0.008821$ \\
\hline & \multicolumn{2}{|c|}{ Estimated $g f$} & $0.00885(5)$ \\
\hline
\end{tabular}


Table $\mathrm{V}$ presents the level specific mass shifts for the isotopic pair ${ }^{4} \mathrm{He} /{ }^{3} \mathrm{He}$ of the $\mathrm{S}$ and $\mathrm{P}^{\circ}$ states arising from configurations $1 \mathrm{~s} 2 l$ and $1 \mathrm{~s} 3 l(l=0,1)$. These results reported by King [31] have been derived from the accurate Hylleraas calculations of Accad et al. [32]. The table shows that the SMS are much larger for the $\mathrm{P}^{\mathrm{o}}$ states than for the $\mathrm{S}$ states and that they have opposite signs for the ${ }^{1} \mathrm{P}^{\mathbf{o}}$ and ${ }^{3} \mathrm{P}^{\mathbf{o}}$ states. This behaviour can be understood qualitatively from a rather simple analysis. Considering the symmetric $(+)$ and antisymmetric (-) spatial parts of the wave functions for the singlet and triplet states respectively,

$\Psi_{ \pm}=\frac{1}{\sqrt{2}}[1 \mathrm{~s}(1) n l(2) \pm n l(1) 1 \mathrm{~s}(2)]$

the \pm sign differentiating the two spin symmetries is reflected in the expectation value of the SMS operator

$\left\langle S_{\mathrm{sms}}\right\rangle_{ \pm}=-\left\langle\Psi_{ \pm}\left|\nabla_{1} \cdot \nabla_{2}\right| \Psi_{ \pm}\right\rangle= \pm \frac{1}{3}\left[J^{1}(1 \mathrm{~s}, n l)\right]^{2} \delta_{l, 1}$

where $J^{1}(1 \mathrm{~s}, n l)$ is the so-called Vinti integral [33]. This, combined with the highly selective Kronecker delta for the one-electron matrix element, explains the above observations. However, the one-configuration approximation is not the entire story. The SMS of the S states are non-zero and those of the ${ }^{1} \mathrm{P}^{o}$ and ${ }^{3} \mathrm{P}^{0}$ states are not identical in absolute value. The expectation value of the specific mass shift operator is indeed highly sensitive to correlation effects, as we will illustrate for the isotope shifts of the $1 s^{2} 2 s^{2} 2 p^{2} P^{o}-1 s^{2} 2 s^{2} 3 s^{2} S$ and $1 s^{2} 2 s^{2} 2 p^{2} P^{o}-1 s^{2} 2 s 2 p^{22} D$ transitions for the ${ }^{11-10} \mathrm{~B}$ isotope pair [34].

In a first step, SD-multireference MCHF expansions (SDMR-MCHF) are generated by considering all single- and double-excitations (SD) from the following reference sets

$\left\{1 \mathrm{~s}^{2} 2 \mathrm{~s}^{2} 2 \mathrm{p}, 1 \mathrm{~s}^{2} 2 \mathrm{p}^{32} \mathrm{P}^{\mathrm{o}}\right\}$,

$\left\{1 s^{2} 2 s^{2} 3 s, 1 s^{2} 2 p^{2} 3 s, 1 s^{2} 2 s 2 p^{2} 2\right\}$

$\left\{1 \mathrm{~s}^{2} 2 \mathrm{~s} 2 \mathrm{p}^{2}, 1 \mathrm{~s}^{2} 2 \mathrm{~s}^{2} 3 \mathrm{~d}^{2} \mathrm{D}\right\}$.

The active set of orbitals is increased up to $n=9 k$. Tripleand quadruple-excitations (TQ) from the same multireference complexes to systematic larger active sets are included afterwards through a series of configuration interaction calculations (SDTQ-MR-CI) using the most elaborate $(n=9 k)$ MCHF radial distributions. These CSF list extensions are specified by using the (SD $[x] \cup \mathrm{TQ}[y])$ notation which represents the union $(\cup)$ of the two CSF sets generated by allowing, from the MR set, single-/double(SD) and triple-/quadruple- (TQ) excitations to the $n=x$ and $n=y$ orbital active sets respectively. The (mass-independent) level SMS expectation values are monitored for the $1 s^{2} 2 s^{2} 2 p^{2} P^{o}$ state in Table VI, when improving the wave function. The difference of the specific mass shift parameters which governs the transition SMS for $1 \mathrm{~s}^{2} 2 \mathrm{~s}^{2} 2 \mathrm{p}^{2} \mathrm{P}_{-}$

Table V. Level specific mass shifts (in $\mathrm{GHz}$ ) of 1 snl ${ }^{1,3} \mathrm{~L}$ for the isotopic pair ${ }^{4} \mathrm{He} /{ }^{3} \mathrm{He}$ (from ref. [30])

\begin{tabular}{llllll}
\hline & $l=0$ & & & $l=1$ \\
\cline { 6 - 6 } \cline { 5 - 6 } Configuration & ${ }^{1} \mathrm{~S}$ & ${ }^{3} \mathrm{~S}$ & & ${ }^{1} \mathrm{P}^{\mathrm{o}}$ & ${ }^{3} \mathrm{P}^{\mathrm{o}}$ \\
\hline $1 \mathrm{~s} 2 l$ & 2.804 & 2.196 & & 13.586 & -19.053 \\
$1 \mathrm{~s} 3 l$ & 0.78 & 0.56 & & 4.293 & -5.420 \\
$1 \mathrm{~s} 4 l$ & 0.32 & 0.219 & & 1.846 & -2.229 \\
\hline
\end{tabular}

Table VI. Specific mass shift expectation values $\left(S_{\text {sms }}\right)$ and total energies (E) for $1 s^{2} 2 s^{2} 2 p^{2} P^{o}$ in $B I$ from $S D-M R-$ $M C H F$ and SDTQ-MR-CI calculations using the $\left\{1 s^{2} 2 s^{2} 2 p\right.$, $\left.1 s^{2} 2 p^{3}\right\}$ reference set (from ref. [34]), for different active sets $(A S)$ with the corresponding number of configuration state functions (NSCF)

\begin{tabular}{lrlr}
\hline AS & $S_{\text {sms }}$ (a.u.) & \multicolumn{1}{l}{$E$ (a.u.) } & NCSF \\
\hline SD-MR-MCHF & & & \\
HF & -0.40486 & -24.529061 & 1 \\
3 & 0.29390 & -24.621891 & 129 \\
4 & 0.24038 & -24.638480 & 520 \\
5 & 0.25662 & -24.645478 & 1301 \\
6 & 0.25443 & -24.648728 & 2584 \\
7 & 0.25327 & -24.650290 & 4479 \\
8 & 0.25212 & -24.651009 & 7096 \\
$9 k$ & 0.25156 & -24.651372 & 10436 \\
SDTQ-MR-CI & & & \\
SD[9k] $\cup$ TQ[3] & 0.26482 & -24.652034 & 10711 \\
SD[9k] $\cup$ TQ[4] & 0.27018 & -24.652687 & 16284 \\
SD[9k] $\cup$ TQ[5] & 0.27159 & -24.652928 & 49134 \\
SD[9k] $\cup$ TQ[6] & 0.27185 & -24.653056 & 99014 \\
\hline
\end{tabular}

$1 s^{2} 2 s^{2} 3 s^{2} S$ is reported in Table VII with the corresponding transition energies. The transition isotope shifts are reported in Table VIII and compared with the Many-Body Perturbation Theory (MBPT) results [36] and with the Fourier Transform Spectroscopy (FTS) measurements [37] for the two transitions. The $2-4 \%$ agreement between theory and experiment is very satisfactory, taking into account that strong destructive interference between NMS and SMS occurs for the $2 \mathrm{p}^{2} \mathrm{P}^{\mathrm{o}}-3 \mathrm{~s}^{2} \mathrm{~S}$ isotope shift.

\subsection{Hyperfine structures in $\mathrm{NaI}$ and $\mathrm{NI}$}

Hyperfine structures are interesting properties for testing the quality of variational wave functions. The theory of hyperfine interaction can be found in [38]. The tensorial form of

Table VII. The difference of the specific mass shift parameters $\left(\Delta S_{s m s}\right)$ and total energies $(\Delta E)$ for the $1 s^{2} 2 s^{2} 2 p^{2} P^{o_{-}}$ $1 s^{2} 2 s^{2} 3 s^{2} S$ transition in $B I$. Also shown are the results from a $S D-M R-C I$ calculation where relativistic shift operators have been added to the non-relativistic Hamiltonian (from ref. [34])

\begin{tabular}{|c|c|c|}
\hline AS & $\Delta S_{\mathrm{sms}}$ (a.u.) & $\Delta E\left(\mathrm{~cm}^{-1}\right)$ \\
\hline \multicolumn{3}{|l|}{ SD-MR-MCHF } \\
\hline $\mathrm{HF}$ & 0.40486 & 38835 \\
\hline 3 & 0.34630 & 40199 \\
\hline 4 & 0.38762 & 39914 \\
\hline 5 & 0.36909 & 39816 \\
\hline 6 & 0.34856 & 39675 \\
\hline 7 & 0.34970 & 39739 \\
\hline 8 & 0.34970 & 39756 \\
\hline $9 \mathrm{k}$ & 0.34976 & 39756 \\
\hline \multicolumn{3}{|c|}{ SD-MR-CI rel. shift } \\
\hline $9 \mathrm{k}$ & & 39738 \\
\hline \multicolumn{3}{|l|}{ SDTQ-MR-CI } \\
\hline $\mathrm{SD}[9 \mathrm{k}] \cup \mathrm{TQ}[3]$ & 0.33677 & 39899 \\
\hline $\mathrm{SD}[9 \mathrm{k}] \cup \mathrm{TQ}[4]$ & 0.33659 & 39973 \\
\hline $\mathrm{SD}[9 \mathrm{k}] \cup \mathrm{TQ}[5]$ & 0.33614 & 40002 \\
\hline $\mathrm{SD}[9 \mathrm{k}] \cup \mathrm{TQ}[6]$ & 0.33629 & 40017 \\
\hline Exp. [35] & & 40039.65 \\
\hline
\end{tabular}


Table VIII. The ${ }^{11} B-{ }^{10} B$ isotope shifts in the $1 s^{2} 2 s^{2} 2 p^{2} P^{o}-1 s^{2} 2 s^{2} 3 s^{2} S$ and $1 s^{2} 2 s^{2} 2 p^{2} P^{o}-1 s^{2} 2 s 2 p^{2}{ }^{2} D$ transitions (from ref. [34]). Contributions from the normal mass shift (nms) and specific mass shift (sms) are separately given

\begin{tabular}{lllll}
\hline Transition & $\mathrm{nms}\left(\mathrm{cm}^{-1}\right)$ & $\mathrm{sms}\left(\mathrm{cm}^{-1}\right)$ & total $\left(\mathrm{cm}^{-1}\right)$ & Method \\
\hline $1 \mathrm{~s}^{2} 2 \mathrm{~s}^{2} 2 \mathrm{p}^{2} \mathrm{P}^{\mathrm{o}}-1 \mathrm{~s}^{2} 2 \mathrm{~s}^{2} 3 \mathrm{~s}^{2} \mathrm{~S}$ & 0.1986 & -0.4409 & -0.2423 & HF [34] \\
& 0.1986 & -0.3661 & -0.1676 & SDTQ-MR-CI [34] \\
& 0.1986 & -0.293 & -0.0944 & MBPT [36] \\
& & & $-0.174 \pm 0.005$ & FTS [37] \\
$1 \mathrm{~s}^{2} 2 \mathrm{~s}^{2} 2 \mathrm{p}^{2} \mathrm{P}^{\mathrm{o}}-1 \mathrm{~s}^{2} 2 \mathrm{~s} 2 \mathrm{p}^{2}{ }^{2} \mathrm{D}$ & 0.2374 & 0.3613 & 0.5987 & HF [34] \\
& 0.2374 & 0.3399 & 0.5773 & SDTQ-MR-CI [34] \\
& & & $0.569 \pm 0.005$ & FTS [37] \\
\hline
\end{tabular}

the hyperfine operators and their matrix elements are given in details by Jönsson et al. [39].

${ }^{23} \mathrm{Na}$ has a nuclear spin $I=3 / 2$ and a nuclear magnetic dipole moment $\mu=2.2176556 \mu_{\mathrm{N}}$. The hyperfine interaction couples the nuclear $\boldsymbol{I}$ and electronic $\boldsymbol{J}$ angular momenta and leads to a splitting from which the magnetic dipole $A_{J}$ and electric quadrupole $B_{J}$ interaction constants can be extracted. We used the active space $\mathrm{MCHF} / \mathrm{CI}$ method for calculating these parameters for the $3 \mathrm{~s}^{2} \mathrm{~S}_{1 / 2}$ and $3 \mathrm{p}^{2} \mathrm{P}_{1 / 2,3 / 2}^{\mathrm{o}}$ levels [15]. The wave functions have been determined through SD-MCHF calculations with the restriction that at most single excitations were allowed from the core. Additional layers of orbitals were optimized for describing the core-core effects, observing the collapse of these correlation orbitals as described in Section 4. Three-particle effects included through CI calculations were found to be of importance for the hyperfine interaction constants. The final values are compared in Table IX with the most accurate experimental values, with the couple cluster (CCSD), relativistic many-body perturbation (RMBPT) and finiteelement multiconfiguration Hartree-Fock (FE-MCHF) theories. The $\mathrm{MCHF} / \mathrm{CI}$ calculated constants all agree to within less than $0.7 \%$ with the experimental values. Combining the $B / Q$ calculated value with the experimental coupling constant, the quadrupole moment $Q=105.6 \mathrm{mb}$ has been obtained, supporting Sundholm and Olsen's conclusion [40] that the uncertainty of muonic values may be larger than expected.

Large-scale MCHF calculations have been performed for $\mathrm{NI}$ an OI [48]. For the $1 \mathrm{~s}^{2} 2 \mathrm{~s}^{2} 2 \mathrm{p}^{3}{ }^{4} \mathrm{~S}_{3 / 2}^{\mathrm{o}}$ nitrogen ground state, with a total orbital angular momentum equal to zero $(L=0, J=S)$, only the Fermi contact term contributes to the hyperfine interaction constant which is given (in $\mathrm{MHz}$ ) by

$A_{J}=95.41067\left(\frac{\mu_{I}}{I}\right)\left(\frac{g_{\mathrm{e}}}{6}\right) \frac{1}{J} a_{\mathrm{c}}$

where

$$
\begin{aligned}
a_{\mathrm{c}}= & \left\langle\gamma L S\left(M_{L}=L\right)\left(M_{S}=S\right)\right| \\
& \times \sum_{i=1}^{N} 8 \pi \delta^{3}\left(\boldsymbol{r}_{i}\right) S_{z i}\left|\gamma L S\left(M_{L}=L\right)\left(M_{S}=S\right)\right\rangle
\end{aligned}
$$

and $g_{\mathrm{e}}=2.0023193$ is the electron spin g-factor. The isotope ${ }^{14} \mathrm{~N}$ has a nuclear spin $I=1$ with a magnetic dipole moment $\mu=0.40376100(6) \mu_{\mathrm{N}}$. In the Hartree-Fock approximation

$\Phi\left(1 \mathrm{~s}^{2} 2 \mathrm{~s}^{2} 2 \mathrm{p}^{3}{ }^{4} \mathrm{~S}^{\mathrm{o}}\right)=\left|1 \mathrm{~s} \overline{\mathrm{s}} 2 \mathrm{~s} \overline{\mathrm{s}} 2 \mathrm{p}_{-1} 2 \mathrm{p}_{0} 2 \mathrm{p}_{+1}\right|$,

the Fermi contact parameter $a_{\mathrm{c}}$ is strictly zero. In this case, the dominating contributions to the hyperfine interaction come from the spin-polarization of the closed $1 \mathrm{~s}$ and $2 \mathrm{~s}$ shells due to the Coulomb exchange interaction with the open shells. Core electrons with the same spin orientation than the $2 p$ electrons are attracted to each other. Two $s$ electrons in the same shell do not have the same density at the nucleus, leading to a net contact interaction [6].

An elegant single-electron picture has been proposed by Chipman $[49,50]$ who suggested to use a compact MCHF variational calculation including the major polarization

Table IX. The interaction constants $A_{J}$ and $B_{J}$ for $3 s^{2} S_{1 / 2}$ and $3 p^{2} P_{1 / 2,3 / 2}^{o}$ states in

\begin{tabular}{|c|c|c|c|c|c|}
\hline \multirow[b]{2}{*}{ Method } & \multirow{2}{*}{$\begin{array}{l}3 \mathrm{~s}^{2} \mathrm{~S} \\
A_{1 / 2}(\mathrm{MHz})\end{array}$} & \multicolumn{3}{|l|}{$3 p^{2} \mathrm{P}^{o}$} & \multirow[b]{2}{*}{ Reference } \\
\hline & & $A_{1 / 2}(\mathrm{MHz})$ & $A_{3 / 2}(\mathrm{MHz})$ & $B_{3 / 2} / Q(\mathrm{MHz} / \mathrm{b})$ & \\
\hline $\mathrm{HF}$ & 626.2 & 63.66 & 12.72 & 15.91 & This work [15] \\
\hline CI & 870.3 & 93.16 & 18.74 & 25.67 & This work [15] \\
\hline $\mathrm{CI}^{\mathrm{a}}$ & 882.2 & 94.04 & 18.80 & 25.79 & This work [15] \\
\hline $\mathrm{CCSD}^{\mathrm{a}}$ & 883.8 & 93.02 & 18.318 & \multirow[t]{3}{*}{26.14} & [41] \\
\hline $\mathrm{RMBPT}^{\mathrm{b}}$ & 860.9 & 91.40 & 19.80 & & {$[42]$} \\
\hline $\mathrm{RMBPT}^{\mathrm{c}}$ & 884.5 & & & & [43] \\
\hline $\mathrm{FE}-\mathrm{MCHF}^{\mathrm{d}}$ & & & & \multirow[t]{3}{*}{25.45} & {$[40]$} \\
\hline \multirow[t]{2}{*}{ Experiment } & $885.8130644(5)$ & 94.42 (19) & $18.69(6)$ & & [44-46] \\
\hline & & & $18.64(6)$ & & [47] \\
\hline
\end{tabular}

${ }^{23} \mathrm{Na}$ compared with values from other calculations and from experiment (from ref. [15])

${ }^{\text {a }}$ Corrected for relativistic effects using the ratio between DF and HF values.

b Third-order calculation.

${ }^{c}$ All order calculation.

${ }^{\mathrm{d}}$ Corrected for relativistic effects using results from quasirelativistic CI calculations. 
effects

$$
\begin{aligned}
& \Phi_{0}=1 \mathrm{~s}^{2} 2 \mathrm{~s}^{2} 2 \mathrm{p}^{3}, \\
& \Phi_{1}=1 \mathrm{ss} * *\left[{ }^{3} \mathrm{~S}\right] 2 \mathrm{~s}^{2} 2 \mathrm{p}^{3}\left[{ }^{4} \mathrm{~S}^{\mathrm{o}}\right], \\
& \Phi_{2}=1 \mathrm{~s}^{2} 2 \mathrm{ss} *\left[{ }^{3} \mathrm{~S}\right] 2 \mathrm{p}^{3}\left[{ }^{4} \mathrm{~S}^{\mathrm{o}}\right], \\
& \Phi_{3}=1 \mathrm{sd}^{* *}\left[{ }^{3} \mathrm{D}\right] 2 \mathrm{~s}^{2} 2 \mathrm{p}^{3}\left[{ }^{2} \mathrm{D}^{\mathrm{o}}\right], \\
& \Phi_{4}=1 \mathrm{~s}^{2} 2 \mathrm{sd} *\left[{ }^{3} \mathrm{D}\right] 2 \mathrm{p}^{3}\left[{ }^{2} \mathrm{D}^{\mathrm{o}}\right] .
\end{aligned}
$$

in which the $\mathrm{s}^{*}$ and $\mathrm{s}^{* *}$ orbitals are orthogonal to $1 \mathrm{~s}$ and $2 \mathrm{~s}$, but are non-orthogonal to each other. Similarly, $d^{*}$ and $d^{* *}$ are two d orbitals, not necessarily orthogonal. Such a nonorthogonal calculation can be done with the "MCHF Atomic Structure Package" (MCHF_ASP) [51] now available from several sites, as described in [52]. The variation principle tailors the correlation orbitals according to the above CSF expansion for describing the 1 s and 2 s spin- and orbital-polarization effects. Figure 6 shows the radial distributions of the $\mathrm{s}^{*}$ and $\mathrm{s}^{* *}$ polarization orbitals. Their shape can be understood by the fact that they can be viewed as representing an entire class of excitations in the conventional configuration interaction picture.

The resulting hyperfine constant $A=7.576 \mathrm{MHz}$, to be compared with the experimental value $A=10.4509 \mathrm{MHz}$ of Hirsch et al. [53], demonstrates that, in comparison to the zero Hartree-Fock result, the single-excitation picture captures polarization effects. However, it is not enough and we need to consider the "true" correlation effects as well. To describe the major correlation effects in the pair-correlation approximation, all single and double (SD) excitations were allowed from the Hartree-Fock reference configuration to the active set of orbitals. The orbital set was then increased in a systematic way, allowing the convergence of the expectation values to be studied. Following the notation used in quantum chemistry, the active set is characterized by the number of orbitals of a certain symmetry. The set $3 \mathrm{~s} 2 \mathrm{p} 1 \mathrm{~d}$ for example, contains three s-orbitals, two p-orbitals and one d-orbital. In Table $\mathbf{X}$ the hyperfine coupling constant, the total energy and the total number of configurations are

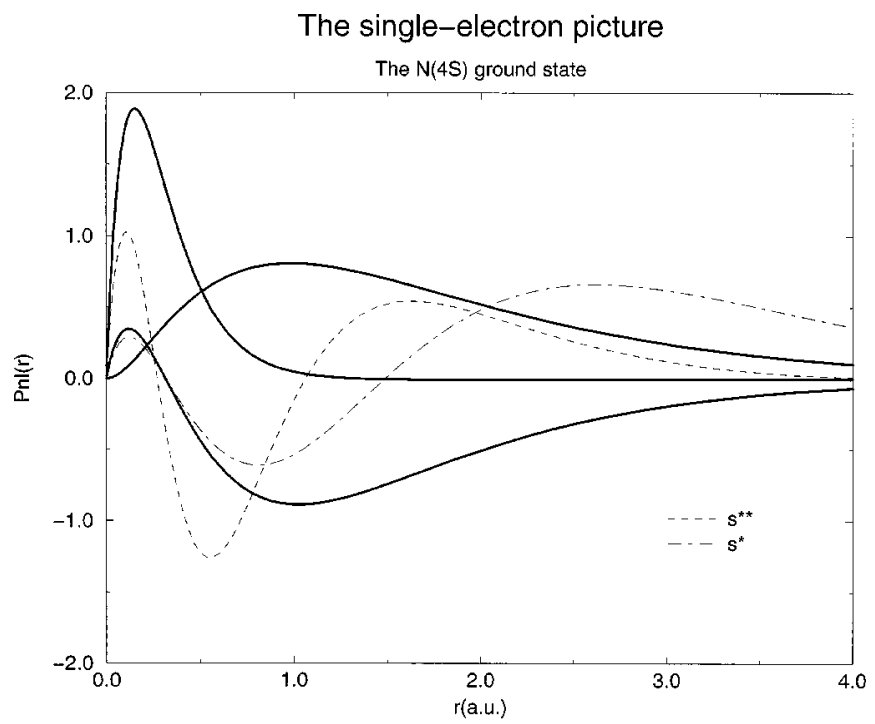

Fig. 6. The single-electron picture. The three thick solid lines correspond to $1 \mathrm{~s}, 2 \mathrm{~s}$ and $2 \mathrm{p}$ orbitals. The shape of the $\mathrm{s}^{*}$ and $\mathrm{s}^{* *}$ polarization orbitals can be understood by the fact that they can be viewed as representing an entire class of excitations in the conventional configuration interaction picture (see text).
Table X. Hyperfine structure constants $(A)$ and total energies (E) for $1 s^{2} 2 s^{2} 2 p^{3}{ }^{4} S^{o}$ in ${ }^{14} N$ (from ref. [48]), for different active sets $(A S)$ with the corresponding number of configuration state functions (NCSF)

\begin{tabular}{lclr}
\hline SD-MCHF & & & \\
\hline AS & $A_{3 / 2}(\mathrm{MHz})$ & $E$ (a.u.) & NCSF \\
\hline HF & 0.0 & -54.400934 & 1 \\
3s2p1d/3s & 8.632 & -54.517526 & 179 \\
4s3p2d1f/3s & 5.267 & -54.558194 & 438 \\
5s4p3d2f1g/3s & 4.924 & -54.571488 & 889 \\
6s5p4d3f2g/3s & 5.592 & -54.576998 & 1538 \\
7s6p5d4f3g/3s & 5.534 & -54.579219 & 2385 \\
8s7p6d5f4g/3s & 5.555 & -54.580233 & 3430 \\
9s8p7d6f5g/3s & 5.595 & -54.580674 & 4673 \\
10s9p8d7f5g/3s & 5.663 & -54.580892 & 5884 \\
\hline SDT- and SDTQ-CI & & & \\
\hline SD $\{10 s 9 p 8 d 7 f 5 g / 3 s\}$ & SDT & & \\
\hline 3s2p1d/3s & 7.488 & -54.582559 & 6511 \\
4s3p2d1f/3s & 9.278 & - & - \\
5s4p3d2f1g/3s & 9.861 & -54.584457 & 20792 \\
6s5p4d3f1g/3s & 10.169 & -54.584689 & 39307 \\
7s6p5d3f1g/3s & 10.234 & -54.584771 & 58878 \\
\hline SD $\{10 s 9 p 8 d 7 f 5 g / 3 s\}$ & $\cup$ SDT\{7s6p5d3f1g/3s $\} \cup$ SDTQ & \\
\hline 4s3p2d1f/3s & 10.395 & -54.585927 & 79373 \\
Experiment [53] & 10.45 & & \\
\hline
\end{tabular}

shown as a function of the increasing active set of orbitals. The three s-orbitals, preceded by a slash in the table, have been added and optimized by allowing single excitations only for describing specifically the spin-polarization effects.

To investigate the influence of higher order correlation effects a number of configuration interaction calculations were performed in which CSFs generated by triple $(\mathrm{T})$ and quadruple $(\mathrm{Q})$ excitations from the reference configuration to the increasing active set were added to the largest expansion from the preceding MCHF calculation. As seen from the table the inclusion of CSFs generated by $\mathrm{T}$ excitations has a drastic effect on the hyperfine coupling constant, which is increased by as much as $80 \%$. The effect of the included CSFs generated by $\mathrm{Q}$ excitations is in comparison very small and configurations obtained by higher excitations are believed to be small and have been neglected. The final result is in rather good agreement with experiment [53].

\section{The relativistic corrections}

The EGAS "logo" that we all have printed on our badge could well have been designed for representing the three forbidden radiative processes connecting the three fine structure levels $2 \mathrm{~s} 2 \mathrm{p}^{3} \mathrm{P}_{J=2,1,0}^{\mathrm{o}}$ and the ground state $2 \mathrm{~s}^{2}{ }^{1} \mathrm{~S}_{0}$ of Be-like ions. The origin of the $2-0$ line is a magnetic quadrupole process (M2). The 1-0 spin-forbidden electric dipole transition (E1]) is induced by the relativistic singlet-triplet mixing while the $0-0$ "strictly forbidden" line can be induced through hyperfine $J$-mixing for isotopes having a non-zero nuclear spin.

As can be seen from Fig. 7 showing the combined spectrum of the planetary nebulae LMC N122 and SMC N2 


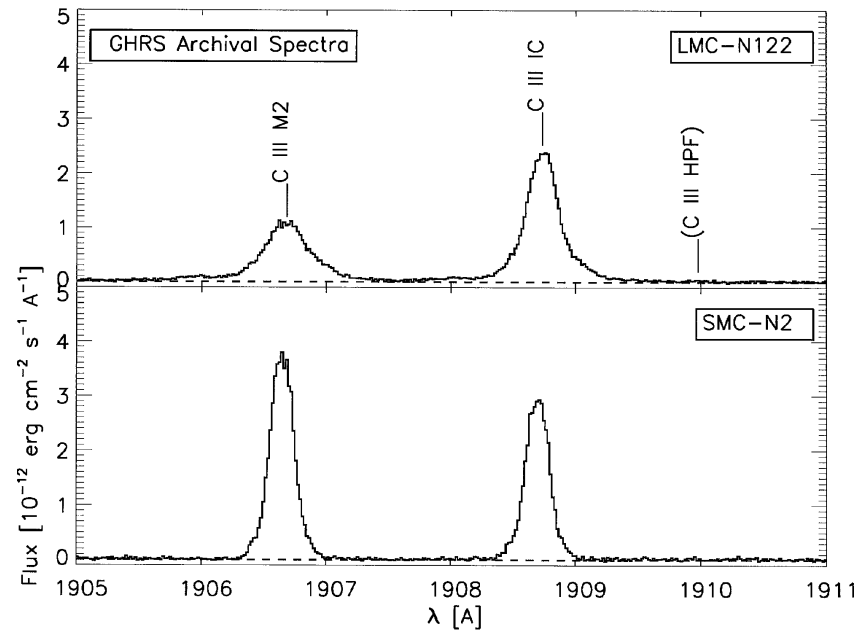

Fig. 7. The combined spectrum of the planetary nebulae LMC N122 and SMC N2 obtained by R. Clegg with the Goddard High Resolution Spectrograph on board on the Hubble Space Telescope.

obtained by R. Clegg with the Goddard High Resolution Spectrograph on board on the Hubble Space Telescope, the relative intensities of the M2 and E1] C III lines are strongly dependent on the source. Their ratio can be used for the determination of the electron density $[54,55]$. The $0-0$ line has been used very recently for measuring the ${ }^{12} \mathrm{C} /{ }^{13} \mathrm{C}$ ratio in planetary nebulae [54] and could be very helpful in the diagnostic of very low-density emitting plasmas [55].

One should realize that relativistic corrections need to be included for the theoretical study of these three processes.

\subsection{The Breit-Pauli approximation}

The one-electron orbital basis (10) optimized using the nonrelativistic MCHF variational approach can be used for building the configuration space in which the relativistic Breit-Pauli (BP) Hamiltonian is diagonalized. This defines the MCHF + BP method. A proper derivation of the BreitPauli approximation is given by Moss [56]. The form of the fine and non-fine structure operators with the relevant matrix elements can be found in [57].

The intermediate coupling eigenvectors,

$\left|\Psi\left(S L J M_{J} \pi\right)\right\rangle=\sum_{i} c_{i}\left|\Phi\left(\alpha_{i} S_{i} L_{i} J M_{J} \pi\right)\right\rangle$

resulting from the diagonalization of the Breit-Pauli Hamiltonian, another configuration interaction problem, takes into account that $J, M_{J}$ and $\pi$ are the only "good" quantum numbers, the operators $\boldsymbol{L}^{2}$ and $\boldsymbol{S}^{2}$ not commuting anymore with the fine-structure operators.

\subsection{The Multiconfiguration Dirac-Fock method}

The multiconfiguration Dirac-Fock (MCDF) method is the fully relativistic counterpart to the non-relativistic $\mathrm{MCHF}$ scheme. Here the Hamiltonian is given by

$\sum_{i=1}^{N}\left[c \boldsymbol{\alpha}_{i} \cdot \boldsymbol{p}_{i}+\left(\beta_{i}-1\right) c^{2}-\frac{Z}{r_{i}}\right]+\sum_{i<j}^{N} \frac{1}{r_{i j}}$,

where $c$ is the speed of light and $\alpha$ and $\beta$ are the Dirac matrices. The atomic wave function is given as an expansion over jj-coupled CSFs

$\left|\Psi\left(J M_{J} \pi\right)\right\rangle=\sum_{i} c_{i}\left|\Phi\left(\alpha_{i} J M_{J} \pi\right)\right\rangle$.
The CSFs are in turn constructed from Slater determinants built on the four-component Dirac orbitals

$\phi(r)=\frac{1}{r}\left(\begin{array}{c}P_{n \kappa}(r) \chi_{\kappa m}(\hat{r}) \\ \mathrm{i} Q_{n \kappa}(r) \chi_{-\kappa m}(\hat{r})\end{array}\right)$.

In the expression above $\kappa$ is the relativistic angular quantum number, $P_{n \kappa}(r)$ and $Q_{n \kappa}(r)$ are the large and small component radial wave functions and $\chi_{\kappa m}(\hat{r})$ is the spinor spherical harmonic in the lsj coupling scheme

$\chi_{\kappa m}(\hat{r})=\sum_{m_{l}, m_{s}}\left\langle l \frac{1}{2} m_{l} m_{s} \mid j m\right\rangle Y_{l m_{l}}(\theta, \varphi) \xi_{m_{s}}(\sigma)$.

Applying the variational condition gives, as in the nonrelativistic case, a set of coupled differential equations for the radial functions together with an eigenvalue equation for the mixing coefficients [58]. These equations are solved to self-consistency. To avoid collapse into the negative energy continua the large and small components of the radial functions need to satisfy additional conditions at the origin [59].

Once a set of radial orbitals has been obtained, relativistic configuration interaction (RCI) calculations can be performed. Here only the expansion coefficients of the CSFs are determined. In the RCI calculations the transverse photon interaction

$$
\begin{aligned}
\mathscr{H}_{\text {trans }}= & -\sum_{i<j}^{N}\left[\frac{\alpha_{i} \cdot \alpha_{j} \cos \left(\omega_{i j} r_{i j}\right)}{r_{i j}}\right. \\
& \left.+\left(\alpha_{i} \cdot \nabla_{i}\right)\left(\alpha_{j} \cdot \nabla_{j}\right) \frac{\cos \left(\omega_{i j} r_{i j}\right)-1}{\omega_{i j}^{2} r_{i j}}\right]
\end{aligned}
$$

as well as the leading QED effects may be included in the Hamiltonian. The photon frequency $\omega_{i j}$ used by the RCI program in calculating the matrix elements of the transverse photon interaction is taken to be the diffence in the diagonal Lagrange multipliers $\varepsilon_{i}$ and $\varepsilon_{j}$ associated with the orbitals. In general, diagonal Lagrange multipliers are approximate electron removal energies only when orbitals are spectroscopic and singly occupied. Thus it is not known how well the MCDF method can determine the full transverse photon interaction when correlation orbitals are present. Frequently, only the low frequency limit $\omega_{i j} \rightarrow 0$, referred to as the Breit interaction, is used. It is this limit that is approximately retained in the $\mathrm{MCHF}+\mathrm{BP}$ approximation.

Fully relativistic calculations are comparatively time consuming. There are several reasons for this. Firstly, the number of radial orbitals is almost twice as large as in the corresponding non-relativistic case. Thus, the active set $\{3 \mathrm{~s}$, $3 \mathrm{p}, 3 \mathrm{~d}\}$ in an MCHF calculation becomes $\left\{3 \mathrm{~s}_{1 / 2}, 3 \mathrm{p}_{1 / 2}\right.$, $\left.3 \mathrm{p}_{3 / 2}, 3 \mathrm{~d}_{3 / 2}, 3 \mathrm{~d}_{5 / 2}\right\}$ in MCDF. Since the CPU time needed to construct and solve the differential equations depend strongly on the number of orbitals, this increase can have large effects. Secondly, the number of CSFs in the atomic wave function expansion is larger in the relativistic case. So does for example the $n=4$ CAS expansions for $1 \mathrm{~s}^{2} 2 \mathrm{p}_{1 / 2}$ and $1 \mathrm{~s}^{2} 2 \mathrm{p}_{3 / 2}$ contain $1048 \mathrm{CSFs}$ whereas the corresponding MCHF expansion for $1 \mathrm{~s}^{2} 2 \mathrm{p}^{2} \mathrm{P}^{\mathrm{o}}$ only contains $205 \mathrm{CSF}$.

Qualitatively relativistic effects manifest in a contraction so that the inner electrons move in orbits closer to the nucleus. This then has an important secondary effect on the more weakly bound relativistic outer electrons [60]. The advantage of the MCDF method compared to the 
$\mathrm{MCHF}+\mathrm{BP}$ is that these effects are described in a direct way through the shape of the radial orbitals. For the $\mathrm{MCHF}+\mathrm{BP}$ method the rearrangement of the electrons is described in a less efficient way through the configuration expansion coefficients. The contraction effects grow rapidly with increasing nuclear charge, and eventually the nonrelativistic orbital basis used in the Breit-Pauli calculation will be completely inadequate to describe the system.

6.3. The spin-forbidden $2 \mathrm{~s} 2 \mathrm{p}^{3} \mathrm{P}_{1}^{\mathrm{o}}-2 \mathrm{~s}^{2}{ }^{1} \mathrm{~S}_{0}$ transition in Be-like ions

The three transitions $2 \mathrm{~s} 2 \mathrm{p}^{3} \mathrm{P}_{J}^{\mathrm{o}}-2 \mathrm{~s}^{2}{ }^{1} \mathrm{~S}_{0}$ have been studied thoroughly along the sequence, using different variational approaches, in a joint effort of combining different approaches to assess the accuracy of the theoretical results. The N IV study [61] is often cited in that sense. The C III (1-0) intercombination line has a very long story [62] and a lot of theoretical effort [63-66] has been made to understand the large decay rate value measured by Kwong et al. [67] using a radiofrequency ion trap. This theoryexperiment persistent discrepancy found a reassuring epilogue in the recent measurements by Doerfert et al. [62] using the heavy-ion storage ring technique, producing a transition probability in perfect agreement with most of the recent variational calculations [68].

The EGAS poster presented by Träbert et al. [69] also reports another successful application of the ion-storage method for measuring the $2 \mathrm{~s} 2 \mathrm{p}^{3} \mathrm{P}_{1}^{\mathrm{o}}$ lifetime in $\mathrm{B}^{+}$, $\tau=(97.65 \pm 1.0) \mathrm{ms}$. Two of the authors of the present publication (MG and CFF) are involved in separate works [70, 71] using both the Breit-Pauli approximation but producing rather different results, $\tau=(103 \pm 2) \mathrm{ms}$ and $\tau=(97.4 \pm 2) \mathrm{ms}$ respectively. We should remember that the theoretical values for these highly sensitive properties are often rescaled in the final step using the observed energy differences affecting the transition rates. This so-called "fine tuning" procedure [72] obviously assumes the use of correct experimental quantities. It appears that the ${ }^{3} \mathrm{P}_{2-0}^{\mathrm{o}}$ energy separation differs following the source. The two sets of calculations would give identical results if using the same fine structure interval which basically governs the strength of the singlet-triplet mixing. This shows that the fine tuning procedure works indeed quite well and that reliable meanlife measurements with a high accuracy could be combined with accurate theoretical line strengths to support a fine structure splitting amongst others.

Intercombination transitions are treated in a very natural way within the relativistic MCDF formalism. Contrary to the MCHF + BP method, where the rate depends critically on an accurate and balanced description of a number of states belonging to different $L S$ terms, only the initial and final states need to be accurately represented. The difficulty with the MCDF method on the other hand is the large and cancelling contributions to the transition matrix element. Due to the large cancellations the convergence of the intercombination rate with respect to the increasing active set is very slow. As an illustration of the MCDF method the $2 s 2{ }^{3} P_{1}^{o}-2 s^{2}{ }^{1} S_{0}$ rate is evaluated for Si XI and Fe XXIII [73]. The configuration expansions were obtained by allowing SD-excitations with at most one excitation from $1 \mathrm{~s}_{1 / 2}$ from, respectively, $\left\{2 \mathrm{~s}_{1 / 2} 2 \mathrm{p}_{1 / 2}, 2 \mathrm{~s}_{1 / 2} 2 \mathrm{p}_{3 / 2}\right\}$ and $\left\{2 \mathrm{~s}_{1 / 2}^{2}\right.$, $\left.2 p_{1 / 2}^{2}, 2 p_{3 / 2}^{2}\right\}$ to the active sets. The Breit interaction has shown to be very important for this transition [74] and was accounted for in subsequent RCI calculations. For the largest expansion additional calculations were performed where now also the frequency dependent part of the transverse photon interaction was included in the Hamiltonian. Finally, the leading QED effect (Lamb shift) was included. The results from these calculations are shown in Table XI. Judging from the convergence patterns the intercombination rates (length form) are believed to be accurate to within less than $1.5 \%$.

\subsection{The $2 \mathrm{~s} 3 \mathrm{p}-2 \mathrm{~s}^{2}$ transitions in Be-like ions}

As a last example, we will illustrate the use of accurate variational calculations in spectroscopy for testing the databased prediction model of Curtis et al. [75] applied to the $2 \mathrm{~s} 3 \mathrm{p}^{1,3} \mathrm{P}_{1}^{\mathrm{o}}-2 \mathrm{~s}^{2}{ }^{1} \mathrm{~S}_{0}$ transitions. The systematization and predictive parametrization of transition probabilties proposed by Curtis and collaborators defines the singlet-triplet

Table XI. Transition energies $(\Delta E)$ and rates $(A)$ for the $2 s 2 p^{3} P_{1}^{o}-2 s^{2}{ }^{1} S_{0}$ intercombination transition (IC) in SiXI and Fe XXIII. Also shown are the length and velocity gf values for the allowed $\left(E_{1}\right) 2 s 2 p^{1} P_{1}^{o}-2 s^{2}{ }^{1} S_{0}$ transition. In the table $[x]$ denotes powers of 10 (from ref. [73])

\begin{tabular}{lllllll}
\hline$A S$ & $\Delta E_{I C}\left(\mathrm{~cm}^{-1}\right)$ & $A_{I C}^{l}\left(s^{-1}\right)$ & $A_{I C}^{v}\left(s^{-1}\right)$ & $\Delta E_{E 1}$ & $g f_{l}$ & $g f_{v}$ \\
\hline Si XI & & & & & & \\
3 & 172680 & $3.396[5]$ & $3.371[5]$ & 332835 & 0.2671 & 0.2630 \\
4 & 172533 & $3.514[5]$ & $3.608[5]$ & 331241 & 0.2660 & 0.2652 \\
5 & 172530 & $3.579[5]$ & $3.888[5]$ & 330633 & 0.2656 & 0.2651 \\
6 & 172536 & $3.606[5]$ & $4.013[5]$ & 330513 & 0.2656 & 0.2651 \\
$B(\omega)$ & 172532 & $3.599[5]$ & $4.016[5]$ & 330508 & 0.2656 & 0.2651 \\
QED & 172176 & $3.577[5]$ & $4.008[5]$ & 330160 & 0.2653 & 0.2654 \\
Exp [16] & 172144 & & & 329679 & & \\
FeXXIII & & & & & & \\
3 & 381619 & $5.143[7]$ & $4.871[7]$ & 758928 & 0.1547 & 0.1520 \\
4 & 381368 & $5.223[7]$ & $5.128[7]$ & 757193 & 0.1544 & 0.1532 \\
5 & 381373 & $5.265[7]$ & $5.337[7]$ & 756570 & 0.1544 & 0.1533 \\
$B(\omega)$ & 382929 & $5.185[7]$ & $5.166[7]$ & 756761 & 0.1546 & 0.1533 \\
QED & 379363 & $5.041[7]$ & $5.118[7]$ & 753366 & 0.1539 & 0.1540 \\
Exp [17] & 379130 & & & 752502 & & \\
\hline
\end{tabular}


"mixing angle" $\theta$ through the trigonometric function involving the energy level data

$\cot (2 \theta) \equiv \pm \frac{1}{\sqrt{2}}\left\{\frac{3\left[E\left({ }^{3} \mathrm{P}_{1}\right)+E\left({ }^{1} \mathrm{P}_{1}\right)-2 E\left({ }^{3} \mathrm{P}_{0}\right)\right]}{2\left[E\left({ }^{3} \mathrm{P}_{2}\right)-E\left({ }^{3} \mathrm{P}_{0}\right)\right]}-1\right\}$.

The "reduced" line strengths for both the resonance and intercombination lines defined from

$S_{i f}^{\mathrm{r}}($ res. $) \equiv S_{i f}($ res. $) / \cos ^{2} \theta$,

$S_{i f}^{\mathrm{r}}\left(\right.$ int.) $\equiv S_{i f}\left(\right.$ int.) $/ \sin ^{2} \theta$

often can be accurately represented by the following isoelectronic behaviour

$Z^{2} S^{\mathrm{r}} \cong a+\frac{b}{Z-c}$

The exposition of measured line strengths in the form of reduced expressions allows to see how well the latter conform to the above linear relationship and possibly make predictions along an isoelectronic sequence.

The details of the MCHF + BP calculations can be found in [28] and [76] for the allowed and spin-forbidden transition respectively. No $L S$ mixing was included for the ground state. For the odd parity, the only $L S$ mixing considered [see eq. (13)] was ${ }^{1,3} \mathrm{P}_{1}^{o}$ mixing in the $J=1$ block. The non-relativistic calculations have already been commented for the allowed transition in BeI (see Section 5.1 and Table IV). Our study in the range of $Z=4-10$ [28] showed the importance of core-valence effects even for $\mathrm{Ne}$ VII on the oscillator strength. Core-core effects are negligible at the $0.2 \%$ level after the first few stages of ionization. The theoretical results [28] support the use of the semi-empirical smoothing method as proposed by Curtis et al. for getting an improvement over experiment. The $\mathrm{F} \mathrm{V}$ ion is probably the most favourable case for which the experimental $2 \mathrm{~s}^{2}{ }^{1} \mathrm{~S}_{0}-2 \mathrm{~s} 3 \mathrm{p}^{1} \mathrm{P}_{1}^{\mathrm{o}}$ transition rate $A=39(6) \mathrm{ns}{ }^{-1}$ deviates substantially from the semi-empirically predicted value (56.2) whereas the latter (with the same error bars) would be in agreement with our variational calculations [62.02(09)].

For the spin-forbidden line, an $(n, n+1)$ optimization procedure was adopted [61] in which the orbitals with principal quantum number $\leqslant n$ were optimized on the ${ }^{3} \mathrm{P}^{0}$ term, and an extra "layer" of orbitals optimized on ${ }^{1} \mathrm{P}^{\mathrm{o}}$ is added to the orbital set. Orbitals for the ground state were optimized separately. Unlike the allowed transition, correlation in the core has not been included. The ${ }^{3} \mathrm{P}^{\mathrm{o}}$ fine structure splitting, which is one of the indicators of accuracy is compared in Table XII with the theoretical full-core plus correlation method (FCPC) values of Zhu and Chung [77] and experiment. In Table XIII the $\mathrm{MCHF}+\mathrm{BP}$ recommended value for each ion is compared with other theory and experiment. Though the agreement is better with experiment for the ion NIV, the present agreement with the semiempirical trend predicted by Curtis et al. [75] is encouraging. The $Z$-expansion results of Ralchenko and Vainshtein [66] are based on perturbation theory. The GRASP results were obtained using a fully relativistic Dirac-Coulomb Hamiltonian with Breit corrections [80] but only valence correlation was considered and the same orbitals were used to describe both the initial and final states. The CIV3 results [81] are based on non-relativistic multiconfiguration expansions built on optimized linear
Table XII. The $2 s 3 p^{3} P_{J}^{o-}{ }^{3} P_{0}^{o}$ fine-structure splitting (in $\left.\mathrm{cm}^{-1}\right)$ (from ref. [76])

\begin{tabular}{|c|c|c|c|c|c|c|}
\hline & B II & C III & N IV & $\mathrm{OV}$ & F VI & $\mathrm{Ne}$ VII \\
\hline \multicolumn{7}{|c|}{ (i) ${ }^{3} \mathrm{P}_{1}^{\mathrm{o}}-{ }^{3} \mathrm{P}_{0}^{\mathrm{o}}$} \\
\hline $\mathrm{MCHF}$ & 1.24 & 5.67 & 16.12 & 36.88 & 73.50 & 133.62 \\
\hline FCPC $^{a}$ & 1.20 & 5.58 & 16.1 & 36.7 & 72.8 & 132.1 \\
\hline Exp. $^{b}$ & 1.30 & 5.67 & 15.83 & 36.7 & 73 & 132.8 \\
\hline \multirow[t]{2}{*}{ Exp. $^{c}$} & & & & & 72 & \\
\hline & (ii) ${ }^{2}$ & ${ }^{3} \mathrm{P}_{0}^{\mathrm{o}}$ & & & & \\
\hline $\mathrm{MCHF}$ & 4.68 & 18.82 & 51.59 & 114.34 & 221.06 & 338.37 \\
\hline $\mathrm{FCPC}^{\mathrm{a}}$ & 4.60 & 18.61 & 51.3 & 113.7 & 219.6 & 385.5 \\
\hline Exp. $^{b}$ & 4.74 & 18.75 & 51.2 & 113.9 & 221 & 388.2 \\
\hline Exp. $^{\mathrm{c}}$ & & & & & 220 & \\
\hline
\end{tabular}

${ }^{\text {a }} \mathrm{Zhu}$ and Chung [77].

b Bashkin and Stoner [35]; Kelly [78].

c Engström [79].

combinations of Slater-type orbitals. As expected, both GRASP and $Z$-expansion results show improving trends as the nuclear charge increases. In Ne VII, the CIV3 values suffer from an overestimate of the ${ }^{3} \mathrm{P}_{1}^{o}-{ }^{1} \mathrm{P}_{1}^{o}$ energy separation used in the final energy level adjustment procedure.

\section{Conclusions}

We hope to have convinced the EGAS audience and the reader of the present paper that the systematic active space $\mathrm{MCHF} / \mathrm{MCDF}$ variational methods, with large multireference CI expansions and efficient CI-algorithms [83, 84], can be helpful in different fields of spectroscopy. With today's powerful workstations, unprecedented accuracy may be achieved for complex systems. The MCHF atomic structure package has been modified for parallel execution on workstations using the parallel virtual machine software [85]. With this modification, MCHF calculations an order of magnitude larger than those of only a few years ago may now be performed. The same trend towards massively parallel applications is expected for the MCDF calculations.

We have shown that the shape of the orbitals resulting from variational calculations strongly depends on the energy functional. This implies that the one-electron basis varies, not only with the model used for tailoring the wave function, but also with the electronic state considered within a given model. A corollary is that a high-quality wave function often demands orbitals optimized for the specific elec-

Table XIII. The Breit-Pauli transition rates $\left(10^{6} s^{-1}\right)$ for the $2 s^{2}{ }^{1} S_{0}-2 s 3 p^{3} P_{1}^{o}$ transition (from ref. [76])

\begin{tabular}{rcccccc}
\hline & & & & & \multicolumn{2}{c}{ Curtis et al. [75] } \\
\cline { 6 - 7 }$Z$ & MCHF & GRASP $^{\mathbf{a}}$ & CIV3 $^{\mathrm{b}}$ & Z-exp $^{\mathrm{c}}$ & Exp. & Pred. \\
\hline 5 & $0.27(6)$ & & & & & 0.1 \\
6 & $0.50(2)$ & & 0.520 & & & 0.4 \\
7 & $3.2(1)$ & 1.40 & 3.07 & 4.97 & $3.3(20)^{\mathrm{d}}$ & 2.8 \\
8 & $19.6(4)$ & 16.4 & 18.3 & 22.2 & $22.6(4)$ & 19 \\
9 & $98.9(9)$ & 85.5 & 88.6 & 95.9 & $102(9)$ & 100 \\
10 & $415(4)$ & 385 & 275 & 370 & $430(60)$ & 418 \\
\hline
\end{tabular}

${ }^{a}$ Fritsche and Grant [80].

${ }^{b}$ Hibbert [81].

${ }^{\mathrm{c}}$ Ralchenko and Vainshtein [66].

${ }^{d}$ Engströn et al. [82]. 
tronic state and, for transition probabilities, it is usually not possible to obtain an accurate description when the same orbital set is used for two different states. The biorthogonal transformation has been implemented for tackling the oneelectron non-orthogonalities resulting from independent optimizations of the initial and final states in the nonrelativistic [86] and relativistic [68] schemes. Alternative procedures using Slater determinant algebra are also explored [87, 88]. More generally, separate optimizations are worthwhile for the calculation of interaction matrix elements in all cases of strong dependence of the one-electron basis with the coupled states considered, whatever the interaction mechanism is. The possible use of the biorthogonal transformation algorithm is currently investigated to calculate the electrostatic coupling between discrete and continuum wave functions in the evaluation of Fano parameters and autoionization rates [89] and the relativistic coupling between separately optimized $L S$ wave functions [90] in Breit-Pauli calculations.

Using large-scale MCHF and CI calculations it is seen that transition probabilities, isotope shifts and hyperfine structures can be calculated very accurately. Higher-order correlation effects beyond the pair-correlation approach are found to be important for the systems considered and need to be included if accurate results are wanted. For transition probabilities, the theoretical calculations are undoubtedly helpful, with an accuracy reaching $0.5 \%$ for the allowed transitions. Spin-forbidden processes are more critical but theory often procedes experiment in determining accurate lifetimes of metastable levels. For isotope shifts, we have shown that the $a b$ initio calculations for light systems for which the specific mass shifts are dominant, can compete with the accuracy of high resolution Fourier transform spectroscopy. The experimental accuracy is unbeatable for hyperfine structure parameters, at least for the ground states for which laser resonance methods can be applied. The comparison theory-experiment is nevertheless interesting, the experimental value guiding the theoretical calculation of the electronic wave functions.

The present paper is limited to transition probabilities, isotope shifts and hyperfine structures but other properties can be evaluated using similar variational approaches. The calculation of negative ions [91], cross sections for ionization of photo-detachment [92], oscillator strength sum rules and dispersion coefficients $[93,94]$ or hyperfine-induced transition rates $[55,95]$ are exciting examples that the reader might enjoy.

The comparison between the Breit-Pauli and Dirac-Fock approaches is important for determining their respective advantages and limits. Effort in this line has been made recently $[96,97]$ but further work is desirable.

The recent methodological and code developments open new perspectives. The Davidson method $[83,84]$ has been implemented recently not only in the MCHF_ASP and GRASP packages, but also in relativistic configurationinteraction codes using finite $B$-spline basis functions [98]. The impact of basis splines in theoretical atomic physics has been evaluated recently in a topical review [99]. Among many successful applications using these techniques, we will point out the model-potential description of Laughlin and Hansen [100] who obtain the "near spectroscopic precision" for the valence spectrum of calcium.
The angular algebra also knows promising developments such as the efficient approach for spin-angular integrations in atomic structure calculations proposed by Gaigalas and Rudzikas [101], using the quasi-spin formalism and the reduced coefficients of fractional parentage. This methodology has been implemented in some codes of the MCHF_ASP package [102].

\section{Acknowledgements}

The authors acknowledge support from the Division of Chemical Sciences, Office of Basic Energy Sciences, Office of Energy Science, U.S. Department of Energy, from the Belgian National Fund for Scientific Reseach (FRFC Convention 2.4551.92) and the French Community of Belgium (Research Convention ARC-93/98-166).

\section{References}

1. Kemble, E. C., "The Fundamental Principles of Quantum Mechanics" (Dover Publications, Inc., New York 1958).

2. Hartree, D. R., Proc. Camb. Phil. Soc. 24, 89 (1927); 27, 111 (1927).

3. Friedrich, H., "Theoretical Atomic Physics" (Sprinter-Verlag, Berlin 1991).

4. Hylleraas, E. and Undheim, B., Z. Phys. 65, 759 (1930).

5. Froese Fischer, C., "The Hartree-Fock Method for Atoms. A Numerical Approach" (John Wiley and Sons, New York 1977).

6. Lindgren, I. and Morrison, J., Atomic Many-Body Theory, "Springer Series in Chemical Physics”, vol. 13 (Springer-Verlag, Berlin 1982).

7. Godefroid, M. R., Froese Fischer, C. and Jönsson, P., Physica Scripta T65, 70 (1996).

8. Yan Zong-Chao and Drake, G. W. F., Phys. Rev. A52, R4316 (1995).

9. Brage, T., Froese Fischer, C. and Jönsson, P., Phys. Rev. A49, 2181 (1994).

10. Volz, U. and Schmoranzer, H., Physica Scripta T65, 48 (1996).

11. Schmitt, A., Volz, U. and Schmoranzer, H., 29th EGAS Meeting (Berlin, 1997), Poster THP76.

12. McAlexander, W. I., Abraham, E. R. I. and Hulet, R. G., Phys. Rev. A54, R5 (1996).

13. Volz, U., Majerus, M., Leibel, H., Schmitt, A. and Schmoranzer, H., Phys. Rev. Lett. 76, 2862 (1996).

14. Oates, C. W., Vogel, K. R. and Hall, J. L., Phys. Rev. Lett. 76, 2866 (1996).

15. Jönsson, P., Ynnerman, A., Froese Fischer, C., Godefroid, M. R. and Olsen, J., Phys. Rev. A53, 4021 (1996).

16. Martin, W. C. and Zalubas, R., J. Phys. Chem. Ref. Data 12, 323 (1983).

17. Shirai, T. et al., J. Phys. Chem. Ref. Data 19, 127 (1990).

18. Curtis, L. J., Beck, R. T. and Ellis, D. G., Phys. Lett. A230, 330 (1997).

19. Froese Fischer, C., J. Phys. B: At. Mol. Phys. 26, 855 (1993).

20. Fleming, J. et al., J. Phys. B: At. Mol. Phys. 29, 4347 (1996).

21. Godefroid, M. R., Olsen, J., Jönsson, P. and Froese Fischer, C., Astrophys. J. 450, 473 (1995).

22. Weiss, A. W., Phys. Rev. A51, 1067 (1995).

23. Fleming, J., Vaeck, N., Hibbert, A., Bell, K. L. and Godefroid, M. R., Physica Scripta 53, 446 (1996).

24. Reistad, N. and Martinson, I., Phys. Rev. A34, 2632 (1986).

25. Träbert, E., Z. Phys. D-At. Mol. Clusters 9, 143 (1988).

26. Sims, J. S. and Whitten, R. C., Phys. Rev. A8, 2220 (1973).

27. Layzer, D., Horak, Z., Lewis, M. N. and Thompson, D. P., Ann. Phys. 29, 101 (1964).

28. Froese Fischer, C., Godefroid, M. R. and Olsen, J., J. Phys. B: At. Mol. Phys. 30, 1163 (1997).

29. Chung, K. T. and Zhu Z.-W., Phys. Rev. A48, 1944 (1993).

30. King, W. H., "Isotope Shifts in Atomic Spectra" (Plenum Press, New York 1984).

31 Bransden, B. H. and Joachain, C. J., "Physics of Atoms and Molecules" (Longman, London and New York 1983), Appendix 8.

32. Accad, Y., Pekeris, C. L. and Schiff, B., Phys. Rev. A4, 516 (1971).

33. Bauche, J. and Champeau, R.-J., Adv. At. Mol. Phys. 12, 39 (1976).

34. Jönsson, P., Froese Fischer, C. and Godefroid, M. R., J. Phys. B: At. Mol. Phys. 29, 2393 (1996). 
35. Bashkin, S. and Stoner, J. O., “Atomic Energy Levels and Grotrian Diagrams", vol. I (Elsevier, New York 1975).

36. Veseth, L., J. Phys. B: At. Mol. Phys. 18, 3463 (1985).

37. Johansson, S. G., Litzen, U., Kasten, J. and Kock, M., Astrophys. J. 403, L25 (1993).

38. Lindgren, I. and Rosén, A., Case Stud. At. Phys. 4, 197 (1974).

39. Jönsson, P., Wahlström, C.-G. and Froese Fischer, C., Comput. Phys. Commun. 74, 399 (1993).

40. Sundholm, D. and Olsen, J., Phys. Rev. Lett. 68, 927 (1992).

41. Salomonson, S. and Ynnerman, A., Phys. Rev. A43, 88 (1991).

42. Johnson, W. R., Idrees, M. and Sapirstein, J., Phys. Rev. A35, 3218 (1987).

43. Liu, Z., Ph.D. Thesis, University of Notre Dame (1989).

44. Beckman, A., Böklen, K. D. and Elke, D., Z. Phys. 270, 173 (1974).

45. Carlsson, J., Jönsson, P., Sturesson, L. and Froese Fischer, C., Physica Scripta 46, 394 (1992).

46. Carlsson, J. and Sturesson, L., Z. Phys. D14, 281 (1989).

47. Krist, Th., Kuske, P., Gaupp, A., Wittmann, W. and Andrä, H. J., Phys. Lett. A61, 94 (1977).

48. Godefroid, M. R., Van Meulebeke, G., Jönsson, P. and Froese Fischer, C., Z. Phys. D42, 193 (1997).

49. Chipman, D. M., Phys. Rev. A39, 475 (1989).

50. Chipman, D. M., J. Chem. Phys. 91, 5455 (1989).

51. Froese Fischer, C., Comput. Phys. Commun. 64, 369 (1991).

52. Froese Fischer, C., Brage, T. and Jönsson, P., "Computational Atomic Structure. An MCHF Approach” (IOP, Bristol 1997).

53. Hirsch, J. M., Zimmerman, G. H. III, Larson, D. J. and Ramsey, N. F., Phys. Rev. A16, 484 (1977).

54. Clegg, R. E. S., Storey, P. J., Walsh, J. R. and Neale, L., Mon. R. Astron. Soc. 284, 348 (1997).

55. Brage, T. et al., Astrophys. J. 500, 507 (1998).

56. Moss, R. R., “Advanced Molecular Quantum Mechanics. An Introduction on Relativistic Quantum Mechanics and the Quantum Theory of Radiation" (Chapman and Hall, London 1973).

57. Hibbert, A., Glass, R. and Froese Fischer, C., Comput. Phys. Commun. 64, 455 (1991).

58. Grant, I. P., "Atomic, and Molecular, \& Optical Physics" (Edited by G. W. F. Drake) (AIP Press, Woodbury, New York 1996), p. 258.

59. Grant, I. P. and Quiney, H. M., Adv. At. Mol. Phys. 23, 37 (1988).

60. Pyykkö, P., Chem. Rev. 88, 563 (1988).

61. Fleming, J. et al., Astrophys. J. 455, 758 (1995).

62. Doerfert, J., Träbert, E., Wolf, A., Schwalm, D. and Uwira, O., Phys. Rev. Lett. 78, 4355 (1997).

63. Fleming, J., Hibbert, A. and Stafford, R. P., Physica Scripta 49, 316 (1994).

64. Froese Fischer, C., Physica Scripta 49, 323 (1994).

65. Ynnerman, A. and Froese Fischer, C., Phys. Rev. A51, 2020 (1995).

66. Ralchenko, Yu. V. and Vainshtein, L. A., Phys. Rev. A52, 2449 (1995).

67. Kwong, V. H. S., Fang, Z., Gibbons, T. T., Parkinson, W. H. and Smith, P. L., Astrophys. J. 411, 431 (1993).

68. Jönsson, P. and Froese Fischer, C., Phys. Rev. A, 57, 4967 (1998).
69. Träbert, E., Doerfert, J., Wolf, A., Linkemann, J. and Tordoir, X., 29th EGAS Meeting (Berlin, 1997), Poster THP073.

70. Fleming, J., Bell, K. L., Hibbert, A., Vaeck, N. and Godefroid, M. R., Mon. Not. R. Astron. Soc. 279, 1289 (1996).

71. Froese Fischer, C. and Gaigalas, G., Physica Scripta 56, 436 (1997).

72. Hibbert, A., Physica Scripa T65, 104 (1996).

73. Jönsson, P., Froese Fischer, C. and Träbert, E., J. Phys. B: At. Mol. Phys. 31, 3497 (1998).

74. Ynnerman, A. and Froese Fischer, C., Phys. Rev. A51, 2020 (1995).

75. Curtis, L. J. et al., Phys. Rev. A51, 4575 (1995).

76. Froese Fischer, C., Gaigalas, G. and Godefroid, M. R., J. Phys. B: At. Mol. Phys. 30, 3333 (1997).

77. Zhu, X.-W. and Chung, K. T., Phys. Rev. A50, 3818 (1994).

78. Kelly, R. L., J. Phys. Chem. Ref. Data 16, 1 (1987).

79. Engström, L., Physica Scripta 31, 379 (1985).

80. Fritzsche, S. and Grant, I. P., Physica Scripta 50, 473 (1994).

81. Hibbert, A., J. Phys. B: At. Mol. Phys. 12, L661 (1979).

82. Engström, L. et al., Physica Scripta 20, 88 (1979).

83. Davidson, E. R., J. Comp. Phys. 17, 87 (1975).

84. Stathopoulos, A. and Froese Fischer, C., Comput. Phys. Commun. 79, 268 (1994).

85. Stathopoulos, A., Ynnerman, A. and Froese Fischer, C., Int. J. Supercomput. Applic. High Perform. Comput. 10, 41 (1996).

86. Olsen, J., Godefroid, M. R., Jönsson, P., Malmqvist, P. A. and Froese Fischer, C., Phys. Rev. E52, 4499 (1995).

87. Fritzsche, S. and Grant, I. P., Comput. Phys. Commun. 92, 111 (1995).

88. Zatsarinny, O., Comput. Phys. Commun. 98, 235 (1996).

89. Vaeck, N. and Godefroid, M. R., work in progress.

90. Olsen, J. and Godefroid, M. R., work in progress.

91. Cowan, R. D., Froese Fischer, C., Hansen, J. E. and Kempter, V., J. Phys. B: At. Mol. Phys. 30, 1457 (1997).

92. Xi, J. and Froese Fischer, C., Phys. Rev. A53, 3169 (1996).

93. Kharchenko, P., Babb, J. F. and Dalgarno, A., Phys. Rev. A55, 3567 (1997).

94. Froese Fischer, C., Jönsson, P. and Godefroid, M. R., Phys. Rev. A57, 1753 (1998).

95. Aboussaïd, A., Godefroid, M. R., Jönsson, P. and Froese Fischer, C., Phys. Rev. A51, 2031 (1995).

96. Fleming, J. and Brage, T., J. Phys. B: At. Mol. Phys. 30, 1385 (1997).

97. Bieroń, J., Jönsson, P. and Froese Fischer, C., 29th EGAS Meeting (Berlin, 1997), Poster FRP001.

98. Chen, M. H. and Cheng, K. T., Phys. Rev. A55, 3440 (1997).

99. Sapirstein, J. and Johnson, W. R., J. Phys. B: At. Mol. Phys. 29, 5213 (1996).

100. Laughlin, C. and Hansen, J. E., J. Phys. B: At. Mol. Phys. 29, L441 (1996).

101. Gaigalas, G. A. and Rudzikas, Z. B., J. Phys. B: At. Mol. Phys. 29, 3303 (1996).

102. Gaigalas, G. A. and Froese Fischer, C., 29th EGAS Meeting (Berlin, 1997), Poster FRP037. 